\title{
On the Memristances, Parameters, and Analysis of the Fractional Order Memristor
}

\author{
Rawid Banchuin (D) \\ Faculty of Engineering and Graduated School of Information Technology, Siam University, Bangkok, Thailand \\ Correspondence should be addressed to Rawid Banchuin; rawid_b@yahoo.com
}

Received 26 July 2018; Revised 5 September 2018; Accepted 24 September 2018; Published 1 November 2018

Academic Editor: Stephan Gift

Copyright (C) 2018 Rawid Banchuin. This is an open access article distributed under the Creative Commons Attribution License, which permits unrestricted use, distribution, and reproduction in any medium, provided the original work is properly cited.

In this work, the analytical expressions of memristances, related parameters, and time domain behavioral analysis of the fractional order memristor have been proposed. Both DC with arbitrary delay and many AC waveforms including arbitrary phase sinusoidal and cosinusoidal waveform along with arbitrary periodic waveform have been taken into account. Unlike the previous works, the formerly ignored dimensional consistency has been taken into account and the analytical modelling of the boundary effect has been performed. Moreover, both transient and asymptotic behaviors of the fractional order memristor excited by AC waveform have been distinguished and analyzed. The effect of phase of AC waveform has also been studied. The influence of the fractional order to the areas of voltage-current hysteresis loop and memristance-current lissajous curve has also been clearly discussed and the usage of fractional order memristor in the memristor based circuit has also been demonstrated.

\section{Introduction}

Recently, a state-of-the-art electrical circuit element, namely, fractional order memristor, is often cited. This circuit element can be obtained from the generalization of the 4th electrical circuit element, namely, memristor, that has been theoretically found by Leon Chua since 1971 [1], by using the concept of fractional calculus which have been adopted in various disciplines, e.g., biomedical engineering [2,3], control system [4-6], and electronic engineering [7-9]. For decades after Chua proposed his original work, the memristor has been practically realized by a research group in Hewlett Packard (HP) labs [10] in 2008. As a result, the mathematical modelling and analysis attempts of the memristor have been proposed (e.g., [11-15]).

For the fractional order memristor on the other hand, there also exists such modelling and analysis attempts [1621]. Some of them generalize the memristor by applying concept of the fractional calculus to the voltage-current relationship [16, 17] and termed such generalized memristor as the fracmemristor [17]. On the other hand, others do so by applying the fractional calculus to the memristor's state equation where the often cited HP memristor has been adopted as the basis [18-21]. However, only the analytical expression of the area of voltage-current hysteresis loop has been proposed in [20] and those of the memristances proposed in $[18,19,21]$ are in terms of the input voltage despite the fact that fractional order memristor of interested is the generalization of the HP memristor which is actually of a charge/current controlled type. Moreover, these previous works also neglected the dimensional consistency $[22,23]$ related issues and the boundary effect, which is an important characteristic of the HP memristor [10], has not been analytically modelled.

By this motivation, we generalize the HP memristor in the fractional order domain by also concerning the formerly ignored dimensional consistency and formulate the analytical expression of memristance in term of the input current where boundary effect has also been modelled. We also derive the expressions of those related parameters of the fractional memristor excited by various exciting waveforms including DC with arbitrary delay and sinusoidal and cosinusoidal with arbitrary phase and arbitrary periodic which are the AC waveforms. With these expressions, parameters, and numerical simulations with MATHEMATICA, the behaviors of the fractional order memristor have been thoroughly explored. Unlike [18-21], both transient and asymptotic behaviors of the fractional order memristor excited by AC 
waveform have been distinguished and analyzed. The effect of phase of AC waveform has also been studied. Moreover, the influence of the fractional order to the areas of voltagecurrent hysteresis loop and memristance-current lissajous curve has been clearly discussed and the usage of fractional order memristor in the memristor based circuit has also been demonstrated.

In the following section, the overview of memristor will be briefly given followed by the memristor's generalization and derivation of our expressions in Section 3 where the behavioral analysis of the fractional order memristor will also be given. The DC waveform will be firstly treated followed by the AC waveforms where the sinusoidal waveform has been emphasized as it is the most fundamental. This is because the memristances and parameters of the memristor excited by the cosinusoidal and arbitrary periodic waveform can be obtained by using those due to the sinusoidal waveform as the basis as will be shown in Section 3 as well. The usage of fractional order memristor in the memristor based circuit will be presented in Section 4 and the conclusion will be finally drawn in Section 5.

\section{The Overview of Memristor}

Memristor is a nonlinear electrical circuit element. This circuit element relates the instantaneous flux, $\phi(t)$, and charge, $q(t)$, through the following relationship:

$$
M(t)=\frac{d \phi(t)}{d q(t)}
$$

where $M(t)$ denotes the memristance.

According to [10], $M(t)$ of the HP memristor can be given in terms of the minimum and maximum values of $M(t)$ denoted by $M_{\text {on }}$ and $M_{\text {off }}$ and the state variable, $x(t)$, as

$$
M(t)=M_{o n} x(t)+(1-x(t)) M_{o f f}
$$

where $x(t)$, which is dimensionless, can be given in terms of the memristor's current, $i(t)$, by

$$
\frac{d x}{d t}=k i(t)
$$

Note that $k=\mu M_{o n} / D^{2}$, where $\mu$ and $D$, respectively, stand for the ion mobility and semiconductor film of thickness. Therefore, the dimension of $k$ is $(\text { Asec })^{-1}$.

As can be seen from (3) and also according to [10], $x(t)$ can be simply given as follows:

$$
x(t)=k q(t)
$$

Therefore, it can be seen that the HP memristor is charge controlled. Since $q(t)$ is a time integration of $i(t)$, it can be stated that the HP memristor is of a current controlled type. Note also that $0 \leq x(t) \leq 1$; thus, $M_{\text {on }} \leq M(t) \leq M_{\text {off }}$ as long as the memristor is unsaturated. Otherwise, $x(t)$ will be bounded at either 0 or 1 so $M(t)$ will be equal to either $M_{\text {on }}$ or $M_{\text {off }}$ according to the boundary effect of the device. Traditionally, such boundary effect can be mathematically modelled by multiplying the RHS of (3) with the window function [24].

\section{The Fractional Order Domain Generalization of the Memristor and the Memristances, Related Parameters, and Analysis of the Fractional Order Memristor}

By generalizing the memristor in the fractional order domain with the fractional calculus, the fractional order memristor can be obtained. Similarly to [18-21], we perform such generalization by applying the fractional calculus to the memristor's state equation, i.e., (3). In these previous works, $d^{\alpha} x / d t^{\alpha}=k i(t)$, where $\alpha$ stands for the order of the fractional order memristor which can be arbitrary real value and has been obtained from such generalization. However, as $x(t)$ is dimensionless; the dimension of the LHS of this previous generalized equation is given by $\sec ^{-\alpha}$ where that of the RHS is $\sec ^{-1}$ which means that a dimensional inconsistency has always existed.

Therefore, the fractional time component [22], $\sigma$, which has the dimension of $\mathrm{sec}$, has been introduced for handling this issue. As a result, unlike [18-21], the following generalized state equation has been used instead.

$$
\sigma^{\alpha-1} \frac{d^{\alpha} x}{d t^{\alpha}}=k i(t)
$$

Similarly to that of the RHS, the dimension of the LHS of (5) is $\sec ^{-1}$; thus the dimensional inconsistency issue has been resolved. Note also that (5) is reduced to (3) when $\alpha=1$ despite the presence of $\sigma$ as $\sigma^{\alpha-1}$ become 1 with such value of $\alpha$.

Unlike $[18,19,21]$, we derive $M(t)$ of the fractional order memristor as a function $i(t)$ as it has been assumed that the of fractional order memristor is a generalization of the HP memristor which is of a current controlled type as aforementioned. Therefore we directly determine $x(t)$ from (5) by using the Riemann-Liouville fractional order integral [25] as follows:

$$
x(t)=x(0)+\frac{k \sigma^{1-\alpha}}{\Gamma(\alpha)} \int_{0}^{t}(t-\tau)^{\alpha-1} i(\tau) d \tau
$$

where $x(0)$ and $\Gamma()$ denote the initial value of $x(t)$ and the Gamma function [26], respectively.

Since it can be seen from (2) that

$$
M(t)=M_{o f f}-M_{d} x(t)
$$

where $M_{d}=M_{o f f}-M_{o n}$, the initial memristance value, i.e., $M(0)$, can be immediately given by

$$
M(0)=M_{o f f}-M_{d} x(0)
$$

Thus by substituting (6) into (7) and keeping (8) in mind, $M(t)$ of the fractional order memristor can be obtained as follows:

$$
M(t)=M(0)-\frac{k \sigma^{1-\alpha} M_{d}}{\Gamma(\alpha)} \int_{0}^{t}(t-\tau)^{\alpha-1} i(\tau) d \tau
$$

which shows that $M(t)$ is current-controlled. 
If we let $\alpha=1,(9)$ will be reduced to

$$
M(t)=M(0)-k M_{d} \int_{0}^{t} i(\tau) d \tau
$$

Since the integer order integration of $i(t)$ gives $q(t)$, we obtain

$$
M(t)=M(0)-k M_{d} q(t)
$$

By using (8), (11) can be simplified under the assumption that $x(0)=0$ and $M_{\text {off }} \gg M_{o n}$ as follows:

$$
M(t)=M_{o f f}(1-k q(t))
$$

which is similar to the original simplified model of the HP memristor [10]. Such correspondence cannot be found in $[18,19,21]$ as the integer order integration of the memristor's voltage, $v(t)$, yields $\phi(t)$.

For traditionally including the boundary effect, the window function must be introduced to the state equation as mentioned above. In [18], the linear window function given by $f(x(t))=1$ has been adopted for simplicity as the usage of more accurate yet more complicated window function; e.g., those Joglekar, Biolek, and Prodomakis [24] can be mathematically cumbersome. Unfortunately, using such linear window function is mathematically equivalent to multiplying the RHS of the state equation by 1 . As a result, no modification has been made on the state equation; thus the boundary effect modelling has not been performed. Moreover, neither the usage of window function nor alternative boundary effect analytical modelling has been made in both [19] and [21].

In order to model the boundary effect in a simplified manner, we apply two mathematical operators, i.e., $\max [x, y]$ and $\min [x, y]$, which, respectively, selects the maximum value and minimum value among $x$ and $y$, to (9). As a result, our expression of $M(t)$ due to arbitrary exciting waveform can be finally given as follows:

$M(t)$

$$
\begin{aligned}
& =\min \left[\max \left[M(0)-\frac{K M_{d}}{\Gamma(\alpha)} \int_{0}^{t}(t-\tau)^{\alpha-1} i(\tau) d \tau, M_{o n}\right],\right. \\
& \left.M_{o f f}\right]
\end{aligned}
$$

where $K=k \sigma^{1-\alpha}$; thus the dimension of $K$ is $\mathrm{A}^{-1} \sec ^{-\alpha}$.

Since the dimension of fractional integral of $i(t)$ is $\operatorname{Asec}^{\alpha}$, that of $\left(K M_{d} / \Gamma(\alpha)\right) \int_{0}^{t}(t-\tau)^{\alpha-1} i(\tau) d \tau$ is given by $\Omega$ which is physically measurable, similarly to those of $M_{d}$ and $M(0)$. Therefore, $M(0)$ and $\left(K M_{d} / \Gamma(\alpha)\right) \int_{0}^{t}(t-\tau)^{\alpha-1} i(\tau) d \tau$, which are at the RHS of (13), can be physically combined as they have the same dimensions and the dimension of $M(t)$, which is the LHS of such equation, has also been found to be such physically measurable $\Omega$; thus it can be seen that our expression of $M(t)$ has dimensional consistency. Moreover, due to the operation of nested $\max [x, y]$ and $\min [x, y], M(t)$ will be equal to $M(0)-\left(K M_{d} / \Gamma(\alpha)\right) \int_{0}^{t}(t-\tau)^{\alpha-1} i(\tau) d \tau$ if and only if $M(0)-\left(K M_{d} / \Gamma(\alpha)\right) \int_{0}^{t}(t-\tau)^{\alpha-1} i(\tau) d \tau$ lies within

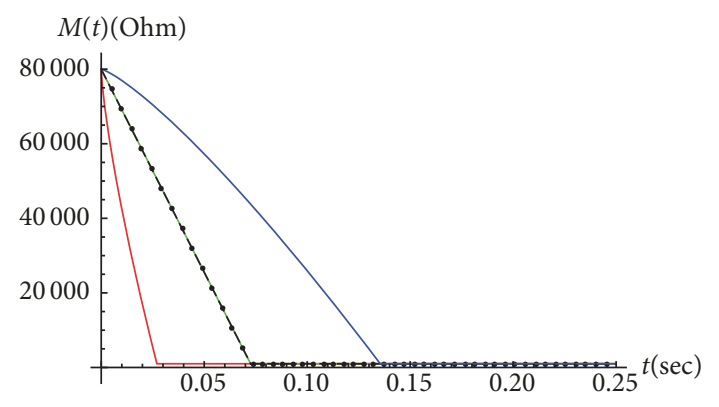

FIGURE 1: $M(t)$ of the fractional order memristor excited by a $110 \mu \mathrm{A}$ DC waveform: $\alpha=0.75$ (red), $\alpha=1$ (green), $\alpha=1.25$ (blue), and HP memristor (black dots).

$\left[M_{o n}, M_{o f f}\right]$ which means that the fractional order memristor remains unsaturated. Otherwise, $M(t)$ will be equal to either $M_{o n}$ or $M_{o f f}$ if $M(0)-\left(K M_{d} / \Gamma(\alpha)\right) \int_{0}^{t}(t-\tau)^{\alpha-1} i(\tau) d \tau$ is lower than $M_{o n}$ or higher than $M_{\text {off }}$ which in turn means that the device become saturated at either its on-state or off-state. Therefore it can be seen that the boundary effect has been modelled without any necessity to use the window function and (13) along with its related results is valid to the saturated fractional order memristor. In the following subsections, $M(t)$ 's due to due to various exciting waveforms and the behavioral analysis of fractional order memristor will be presented.

3.1. DC Waveform. Mathematically, the DC waveform with arbitrary delay $\left(t_{d}\right)$, which is more generic than the undelay waveform assumed in the previous works [18, 19], can be defined as $i(t)=I_{D C} u\left(t-t_{d}\right)$, where $I_{D C}$ and $u(t)$ denote the magnitude of the waveform and the unit step function. Therefore, the resulting $M(t)$ can be straightforwardly obtained by using (13) as

$M(t)$

$$
\begin{aligned}
& =\min \left[\max \left[M(0)-\frac{K M_{d} I_{D C}\left(t-t_{d}\right)^{\alpha}}{\Gamma(\alpha+1)}, M_{o n}\right],\right. \\
& \left.M_{o f f}\right]
\end{aligned}
$$

By using (14) with $t_{d}=0 \mathrm{sec}, K=100000 \mathrm{~A}^{-1} \sec ^{-\alpha}, M_{\text {on }}=1$ $\mathrm{k} \Omega, M_{o f f}=100 \mathrm{k} \Omega$, and $M(0)=80 \mathrm{k} \Omega, M(t)$ 's of the fractional order memristor with various $\alpha$ 's excited by the DC waveform can be numerically simulated as depicted in Figures 1 and 2 where $I_{D C}=110 \mu \mathrm{A}$ and $I_{D C}=-110 \mu \mathrm{A}$ have been, respectively, assumed and $M(t)$ 's of the HP memristor simulated by using its SPICE model [27] have also been included.

From these figures, the strong agreements between our $M(t)$ 's obtained by using (14) with $\alpha=1$ and those of the HP memristor can be observed. Since the HP memristor is of order 1 in the context of fractional order domain, such strong agreements verify our expression. These figures also show that $M(t)$ of the fractional order memristor can be either the increasing or decreasing function of $t$ with the final value of 


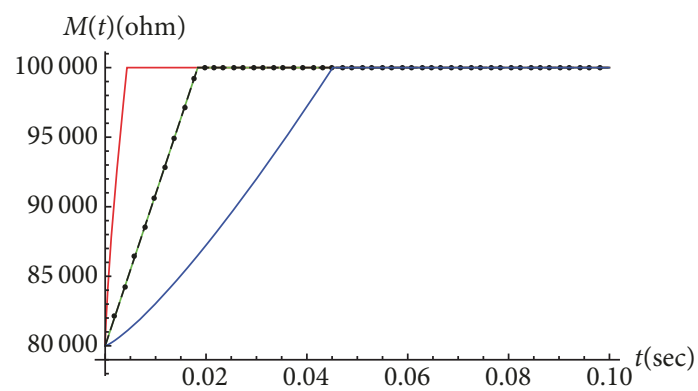

FIgURE 2: $M(t)$ of the fractional order memristor excited by a -110 $\mu \mathrm{A}$ DC waveform: $\alpha=0.75$ (red), $\alpha=1$ (green), $\alpha=1.25$ (blue), and HP memristor (black dots).

$M_{\text {on }}$ or $M_{\text {off }}$ when the fractional order memristor become saturated if we let $I_{D C}>0$ or $I_{D C}<0$. Moreover, the rate of change of $M(t)$ has been found to be inversely proportional to $\alpha$.

Since it can be seen that the fractional order memristor become saturated at a certain time given by $t_{\text {sat }}$, by using (14), we have

$$
M(0)-\frac{K M_{d} I_{D C}\left(t_{s a t}-t_{d}\right)^{\alpha}}{\Gamma(\alpha+1)}= \begin{cases}M_{o n} & I_{D C}>0 \\ M_{o f f} & I_{D C}<0\end{cases}
$$

As a result, $t_{\text {sat }}$ can be immediately given as follows:

$$
\begin{aligned}
& t_{\text {sat }} \\
& = \begin{cases}t_{d}+\left[\frac{\Gamma(\alpha+1)\left(M(0)-M_{o n}\right)}{k M_{d} I_{D C}}\right]^{1 / \alpha} & I_{D C}>0 \\
t_{d}+\left[\frac{\Gamma(\alpha+1)\left(M(0)-M_{o f f}\right)}{k M_{d} I_{D C}}\right]^{1 / \alpha} & I_{D C}<0\end{cases}
\end{aligned}
$$

which shows that $t_{\text {sat }}$ is directly proportional to the size of the difference between $M(0)$ and $M_{o n}\left(M_{o f f}\right)$. So, $t_{\text {sat }}$ reaches its maximum value, i.e., $t_{\text {sat }(M A X)}$, if and only if $M(0)$ reaches its possible peak value given by either $M_{\text {off }}$ when $I_{D C}>0$ or $M_{\text {on }}$ when $I_{D C}<0$. Thus $t_{\text {sat (MAX) }}$ can be found as

$$
t_{\text {sat }(M A X)}= \begin{cases}t_{d}+\left[\frac{\Gamma(\alpha+1)}{K I_{D C}}\right]^{1 / \alpha} & I_{D C}>0 \\ t_{d}+\left[\frac{\Gamma(\alpha+1)}{K\left(-I_{D C}\right)}\right]^{1 / \alpha} & I_{D C}<0\end{cases}
$$

which can be immediately given in a more compact manner as follows:

$$
t_{s a t(M A X)}=t_{d}+\left[\frac{\Gamma(\alpha+1)}{K\left|I_{D C}\right|}\right]^{1 / \alpha}
$$

For confirming the aforesaid observation on the relationship between the rate of change of $M(t)$ and $\alpha, t_{\text {sat (MAX) }}$ 's have been simulated by using (18) under the similar assumptions to those of the simulation of $M(t)$ 's shown in Figures 1 and 2 but with varying $I_{D C}$, as depicted in Figure 3 which shows that $t_{\text {sat (MAX) }}$ is directly proportional to $\alpha$. Due to the definition of $t_{\text {sat }(M A X)}$, this confirms such observation. Moreover, it can also be seen from Figure 3 that $t_{\text {sat (MAX) }}$ is inversely proportional to $\left|I_{D C}\right|$.

3.2. AC Waveforms. Among various AC waveforms, the sinusoidal waveform has been emphasized as it is the foundation of the others. Unlike those previous works, the sinusoidal waveform with arbitrary phase $(\theta)$ has been chosen due to its generality. Mathematically, such waveform can be given by $i(t)=I_{m} \sin (\omega t+\theta)$, where $I_{m}$ and $\omega$, respectively, denote the peak value and angular frequency of $i(t)$. By using (13), $M(t)$ of the fractional order memristor under the arbitrary phase sinusoidal input can be given by

$M(t)$

$$
\begin{aligned}
& =\min \left[\max \left[M(0)-\frac{K M_{d} I_{m} t^{\alpha}}{\Gamma(\alpha+1)}\left[\sin (\theta){ }_{1} \mathrm{~F}_{2}\left(1 ; \frac{\alpha}{2}+\frac{1}{2}, \frac{\alpha}{2}+1 ;-\frac{1}{4}(\omega t)^{2}\right)+\frac{\omega t \cos (\theta)}{\alpha+1}{ }_{1} \mathrm{~F}_{2}\left(1 ; \frac{\alpha}{2}+1, \frac{\alpha}{2}+\frac{3}{2} ;-\frac{1}{4}(\omega t)^{2}\right)\right], M_{\text {on }}\right],\right. \\
& \left.M_{\text {off }}\right]
\end{aligned}
$$

where ${ }_{1} \mathrm{~F}_{2}(;, ;$; ) denotes a generalized hypergeometric function with $p=1$ and $q=2$ [28].

By letting $K=100000 \mathrm{~A}^{-1} \sec ^{-\alpha}, M_{\text {on }}=1 \mathrm{k} \Omega, M_{\text {off }}=100$ $\mathrm{k} \Omega$, and $M(0)=80 \mathrm{k} \Omega$ similarly to the previous subsection, $M(t)$ 's of the fractional order memristor with various $\alpha$ 's excited by the sinusoidal input can be simulated as depicted in Figures 4 and 5, where $I_{m}=110 \mu \mathrm{A}$ and $I_{m}=-110 \mu \mathrm{A}$ have been, respectively, assumed and $M(t)$ 's of the HP memristor simulated by using its SPICE model have also been included. Moreover, we also let $\omega=1 \mathrm{rad} / \mathrm{sec}$ and $\theta=0 \mathrm{rad}$. Again, the strong agreements between our expression based $M(t)$ 's with $\alpha=1$ and those of the HP memristor which verify our expression can be observed. It can be seen that $M(t)$ of the fractional order memristor with $\alpha \leq 1$ is periodic with clipped peaks at $M_{o n}$ and $M_{o f f}$ due to the temporary saturation of the fractional order memristor as the unclipped peaks of $M(t)$ lie outside $\left[M_{o n}, M_{o f f}\right]$. On the other hand, $M(t)$ of the device with $\alpha>1$ is not periodic but time independently equal to either $M_{\text {on }}$ or $M_{\text {off }}$ when $t \geq T_{\text {sat }}$ up to the sign of $I_{m}$. This is because the device becomes 


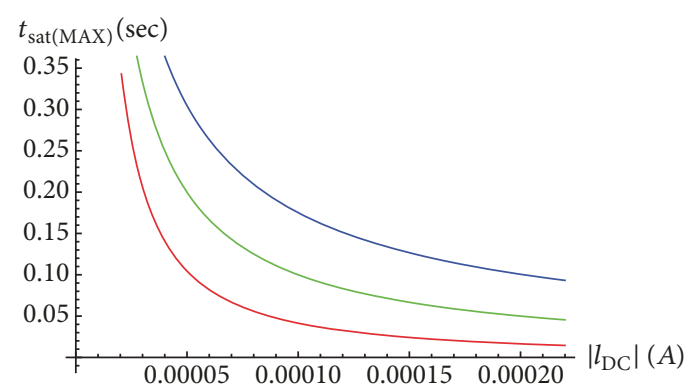

FIGURE 3: $t_{\text {sat(MAX) }}$ of the current-controlled fractional order memristor against $\left|I_{D C}\right|: \alpha=0.75$ (red), $\alpha=1$ (green), and $\alpha=1.25$ (blue).

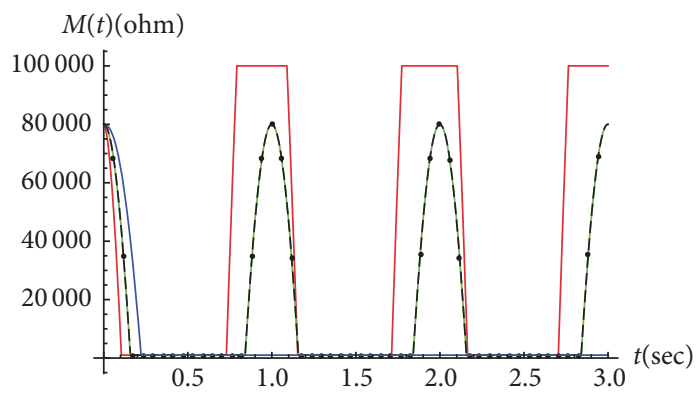

Figure 4: $M(t)$ of the fractional order memristor under the sinusoidal input with $I_{m}=110 \mu \mathrm{A}: \alpha=0.75$ (red), $\alpha=1$ (green), $\alpha=1.25$ (blue), and HP memristor (black dots).

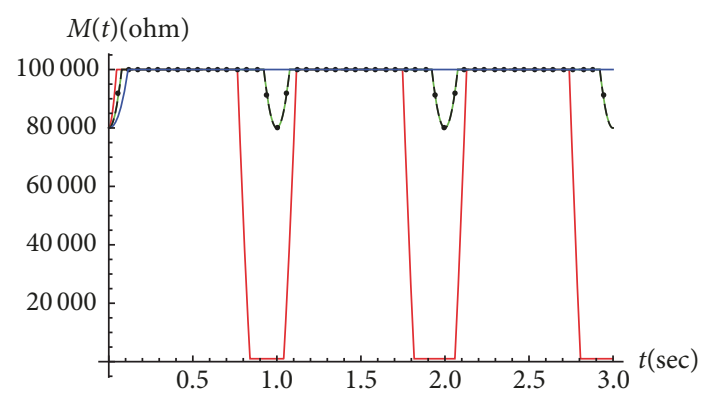

FIGURE 5: $M(t)$ of the fractional order memristor under the sinusoidal input with $I_{m}=-110 \mu \mathrm{A}: \alpha=0.75$ (red), $\alpha=1$ (green), $\alpha=1.25$ (blue), and HP memristor (black dots).

permanently saturated after $T_{\text {sat }}$ as $M(t)$ contains the time proportional term which starts to lie outside $\left[M_{o n}, M_{\text {off }}\right]$ at $t=T_{\text {sat }}$ when $\alpha>1$. In order to show that the permanent saturation of the fractional order memristor with $\alpha>1$ excited by sinusoidal input is possible, we simulate $M(t)$ of the device with $\alpha=1.25$ once again by using (20) which is (19) without the boundary effect. The simulation results have been depicted in Figures 6 and 7 which show that $M(t)$ 's eventually lie outside $\left[M_{o n}, M_{o f f}\right]$ permanently. This yields the saturation when such effect has been included.

$$
\begin{aligned}
& M(t)=M(0)-\frac{K M_{d} I_{m} t^{\alpha}}{\Gamma(\alpha+1)}[\sin (\theta) \\
& \cdot{ }_{1} \mathrm{~F}_{2}\left(1 ; \frac{\alpha}{2}+\frac{1}{2}, \frac{\alpha}{2}+1 ;-\frac{1}{4}(\omega t)^{2}\right)+\frac{\omega t \cos (\theta)}{\alpha+1}
\end{aligned}
$$

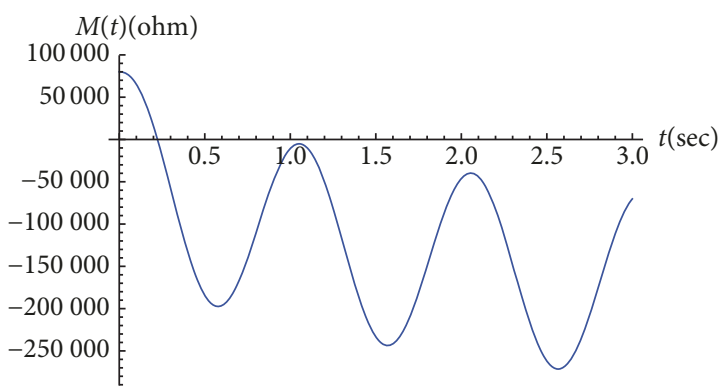

FIGURE 6: $M(t)$ of the boundary effect free fractional order memristor under the sinusoidal input with $I_{m}=110 \mu \mathrm{A}$ and $\alpha=1.25$.

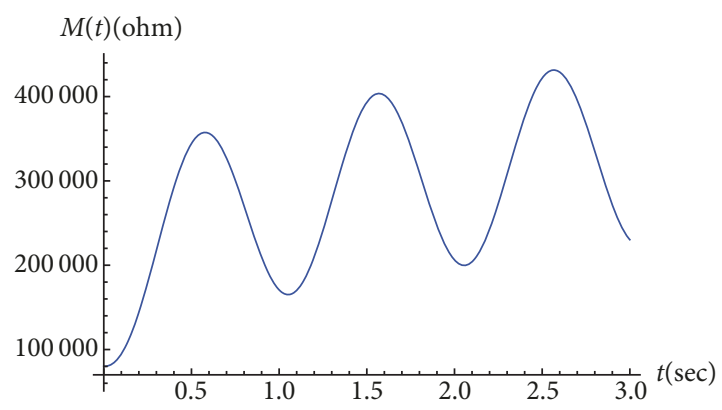

FIGURE 7: $M(t)$ of the boundary effect free fractional order memristor under the sinusoidal input with $I_{m}=-110 \mu \mathrm{A}$ and $\alpha=1.25$.

$$
\left.\cdot{ }_{1} \mathrm{~F}_{2}\left(1 ; \frac{\alpha}{2}+1, \frac{\alpha}{2}+\frac{3}{2} ;-\frac{1}{4}(\omega t)^{2}\right)\right]
$$

However, it should be mentioned here that both temporary and permanent saturation do not always occur. Instead, their occurrences are dependent on the conditions on parameters, which makes either the peaks or time proportional term of $M(t)$ be outside $\left[M_{o n}, M_{o f f}\right]$ such as those of Figures 4 and 5. Moreover the determination of such time proportional term and $T_{\text {sat }}$ will be presented later in this subsection.

Similarly to the memristor, the voltage-current lissajous curve is of interest for the fractional order memristor. Therefore, those curves of the fractional order memristor will be simulated by using (19) as the basis for further studying the behavior of this device under the AC input. Now, we let $0 \mathrm{sec}<t \leq 19 \mathrm{sec}, \omega=1 \mathrm{rad} / \mathrm{sec}$, and $\theta=0 \mathrm{rad}$; the lissajous curves of the fractional order memristor with various $\alpha$ 's can be simulated as depicted in Figures 8-13 where $I_{m}=110 \mu \mathrm{A}, K=10000 \mathrm{~A}^{-1} \mathrm{sec}^{-\alpha}, M_{o n}=100 \Omega, M_{\text {off }}=16 \mathrm{k} \Omega$, and $M(0)=11 \mathrm{k} \Omega$ have been assumed in Figures 8-10. On the other hand, $I_{m}=-150 \mu \mathrm{A}, K=10000 \mathrm{~A}^{-1} \mathrm{sec}^{-\alpha}, M_{o n}=$ $100 \Omega, M_{\text {off }}=38 \mathrm{k} \Omega$, and $M(0)=11.2 \mathrm{k} \Omega$ have been adopted in Figures 11-13. Based on its SPICE model, the lissajous curves of the HP memristor have also been simulated and compared to those of the fractional order memristor with $\alpha=1$ as depicted in Figures 9 and 12 where the strong agreements between the fractional order memristor and HP memristor based curves which are both time invariant shaped 


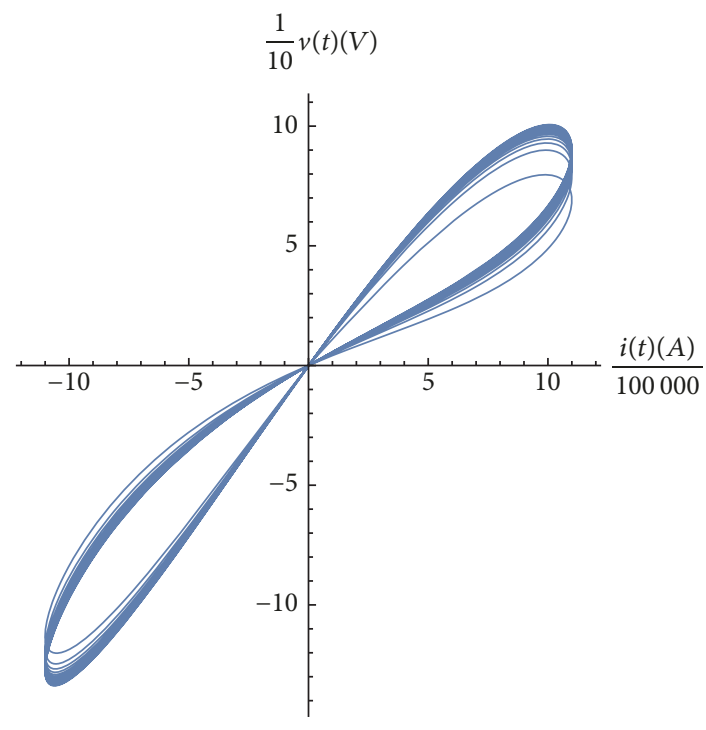

Figure 8: $v(t)-i(t)$ of the fractional order memristor: $\alpha=0.75$ and $I_{m}>0$.

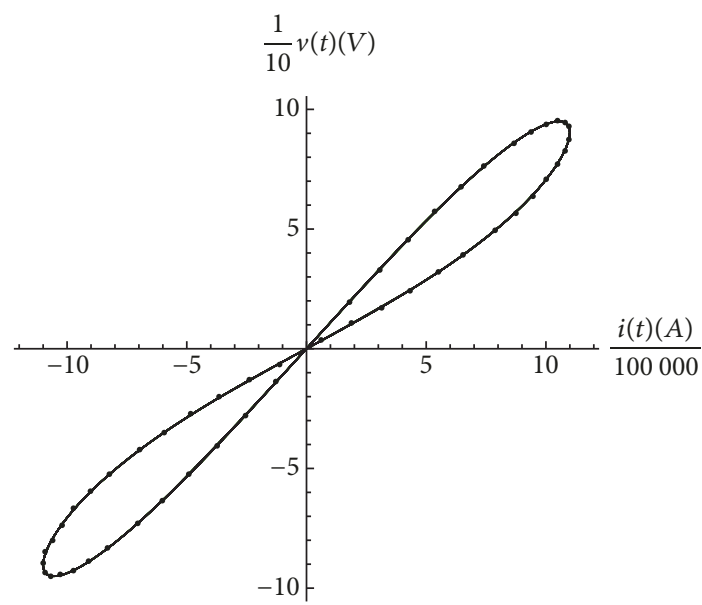

Figure 9: $v(t)-i(t)$ of the fractional order memristor (green) and HP memristor (black dots): $\alpha=1$ and $I_{m}>0$.

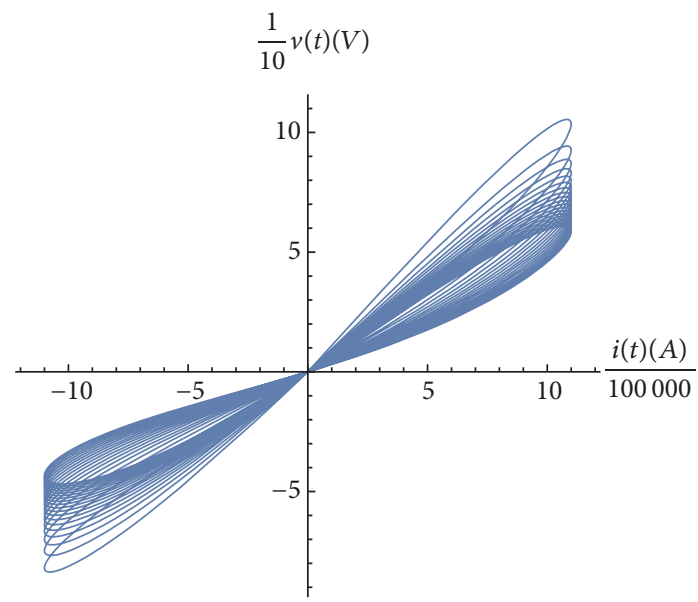

FIGURE 10: $v(t)-i(t)$ of the fractional order memristor: $\alpha=1.25$ and $I_{m}>0$.

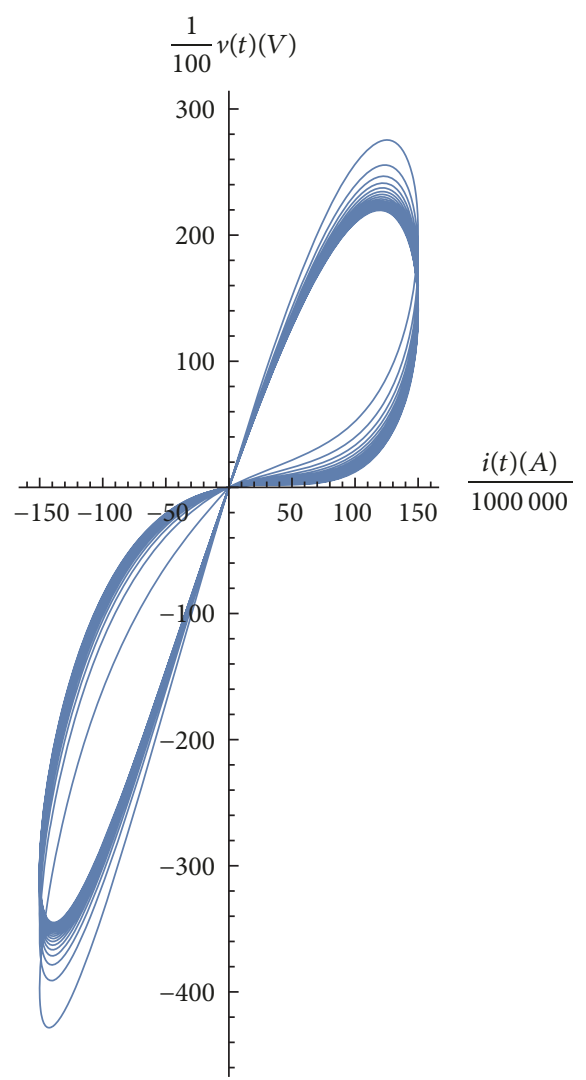

FIgURE 11: $v(t)-i(t)$ of the fractional order memristor: $\alpha=0.75$ and $I_{m}<0$.

symmetric pinched hysteresis loops can be observed. Again, this verifies the accuracy of our expression.

From other figures, it can be seen that the lissajous curve of the fractional order memristor with $\alpha \neq 1$ also takes the pinched hysteresis loop shape with pinching point at the origin despite the asymmetricities which means that the fractional order memristor preserves the memristive characteristic [30]. This is unlike those fracmemristor based results previously proposed in [17] which do not display the pinched hysteresis loop at all; therefore such fracmemristor does not employ the memristive characteristic according to [30]. Unlike those of the fractional order memristor with $\alpha=1$ and HP memristor, the shape of the lissajous curve of the fractional order memristor with $\alpha \neq 1$ keeps changing. This is because $M(t)$ of such fractional order memristor contains the time proportional term. This can be clearly seen from Figures 14-17 which display $M(t)$ 's of the fractional order memristor with $\alpha \neq 1$. Unlike the previous ones depicted in Figures 4 and 5, the peaks of these $M(t)$ 's with $\alpha<1$ are unclipped as can be seen from Figures 14 and 16. This is because fractional order memristor is always unsaturated as such peaks lie within $\left[M_{o n}, M_{o f f}\right]$. Moreover, the fractional order memristor with $\alpha>1$ never becomes saturated and can be seen from Figures 15 and 17. This is because the time proportional terms of its $M(t)$ 's are always be within $\left[M_{o n}, M_{o f f}\right]$. At this point, it can be seen that both temporary and permanent saturation of the fractional order 


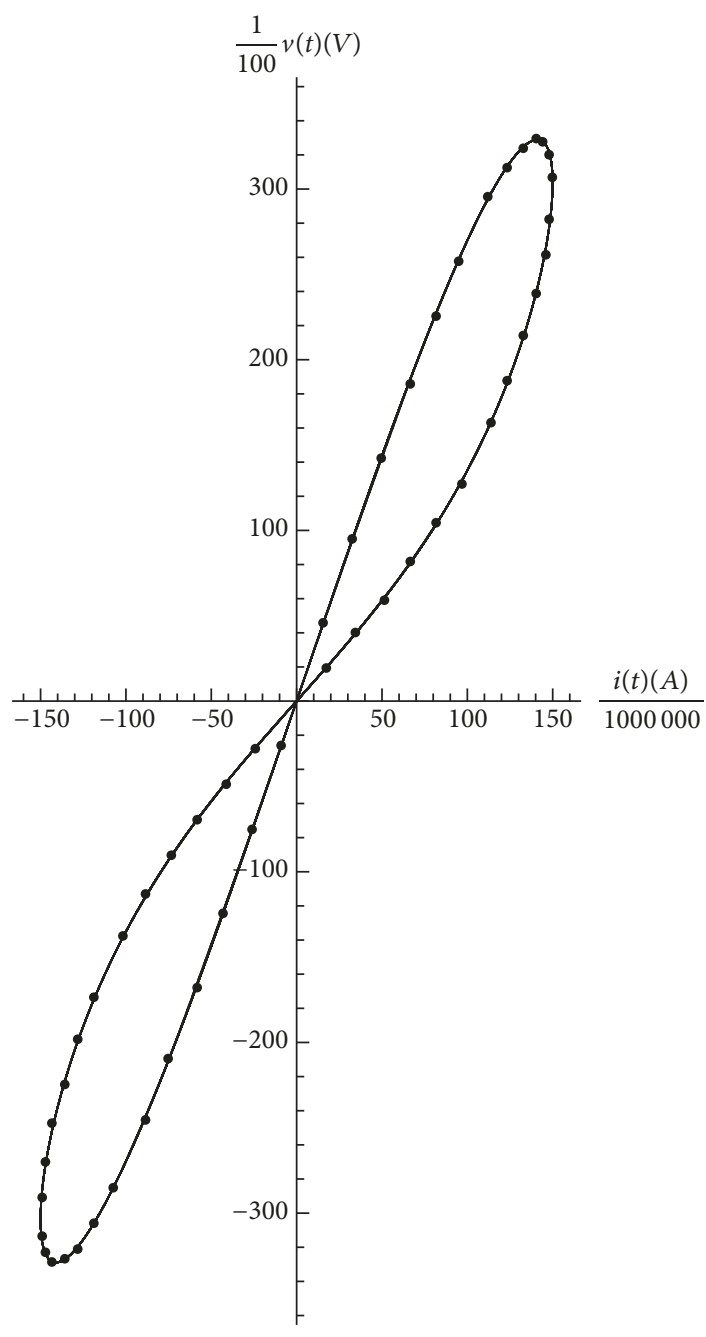

FIGURE 12: $v(t)-i(t)$ of the fractional order memristor (green) and HP memristor (black dots): $\alpha=1$ and $I_{m}<0$.

memristor under sinusoidal excitation do not always occur but depend on the conditions on parameters and input as aforementioned.

At asymptotic state, it can be seen that such time proportional term of $M(t)$ becomes time independent instead. Therefore, the shape of its lissajous curves of the fractional order memristor with $\alpha \neq 1$ is asymptotically unchanged as can be seen from Figures 18 and 19 which depict the lissajous curves of the fractional order memristor with various $\alpha$ 's simulated by assuming that $100 \mathrm{sec}<t \leq 119 \mathrm{sec}$. Apart from being asymmetric when $\alpha \neq 1$, we have found that the lobe area of the lissajous curve of the fractional order memristor is affected by $\alpha$ similarly to those proposed in $[20,21]$. In particular, we have found that the fractional order memristor with lower $\alpha$ yields the lissajous curve with wider lobe area which refers to more memory effect and less linearity. Besides the voltage-current curve, the memristancecurrent lissajous curve has been found to be also interesting. Therefore, such curves of the fractional order memristor with various $\alpha$ 's have also been simulated as depicted in Figures

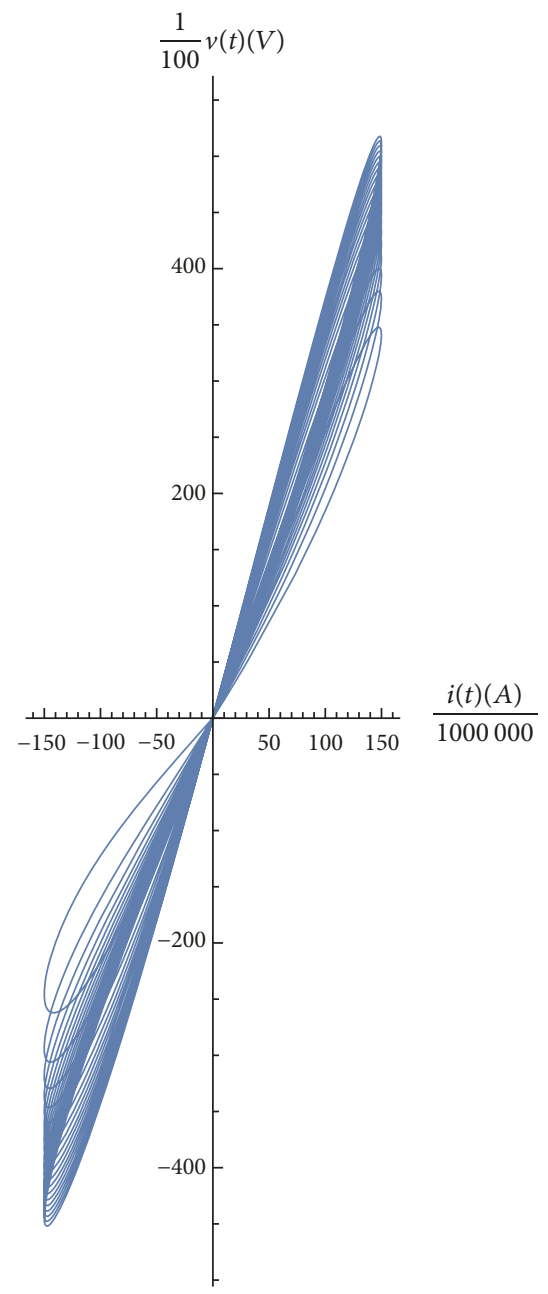

FIGURE 13: $v(t)-i(t)$ of the fractional order memristor: $\alpha=1.25$ and $I_{m}<0$.

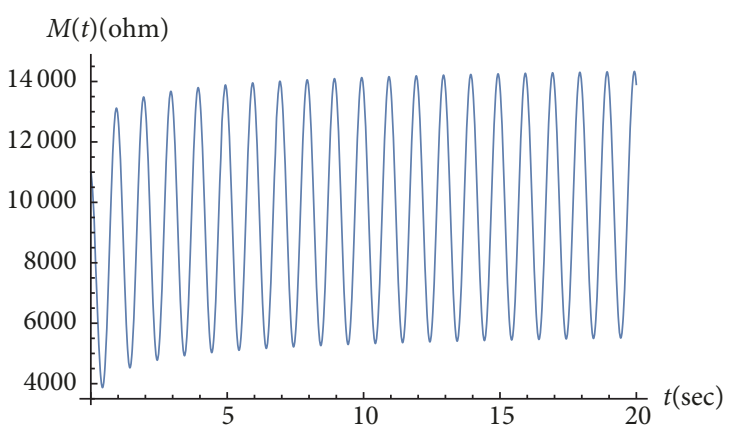

FIGURE 14: $M(t)$ of the fractional order memristor under the sinusoidal input with $I_{m}>0: \alpha=0.75$.

20 and 21 where the parameters setting similar to those of the voltage-current curve have been assumed. Moreover, we also assume that $100 \mathrm{sec}<t \leq 119 \mathrm{sec}$. These figures show that the resulting lissajous curves take the elliptical closed loop shape which are unchanged as $M(t)$ enters the asymptotic state at the assumed time interval. The elliptical shaped lissajous curve means that $M(t)$ is periodic as well as 


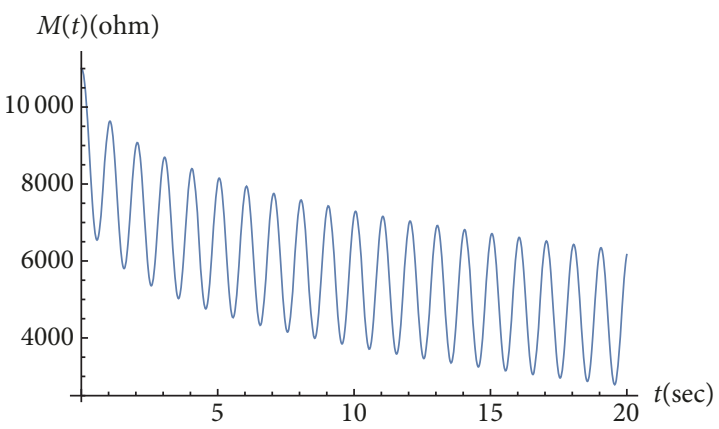

FIGURE 15: $M(t)$ of the fractional order memristor under the sinusoidal input with $I_{m}>0: \alpha=1.25$.

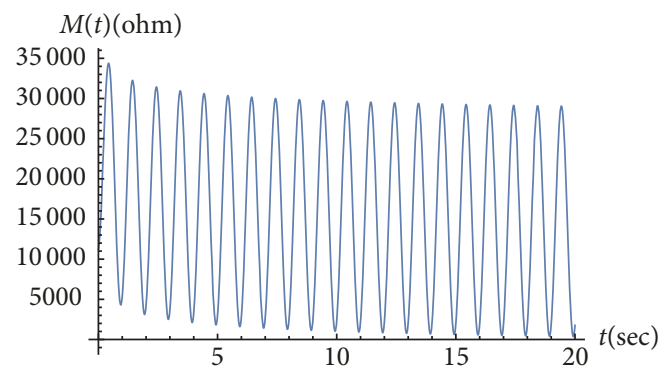

FIGURE 16: $M(t)$ of the fractional order memristor under the sinusoidal input with $I_{m}<0: \alpha=0.75$.

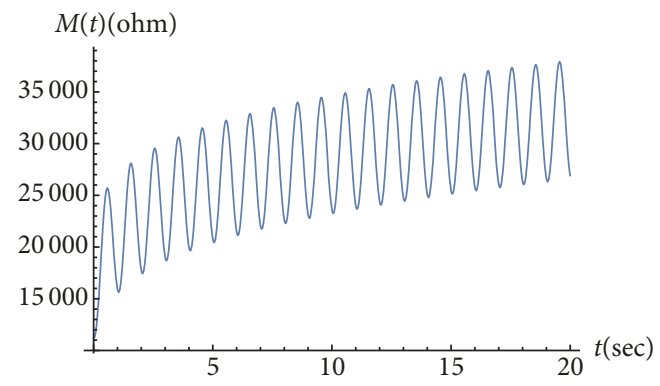

FIGURE 17: $M(t)$ of the fractional order memristor under the sinusoidal input with $I_{m}<0: \alpha=1.25$.

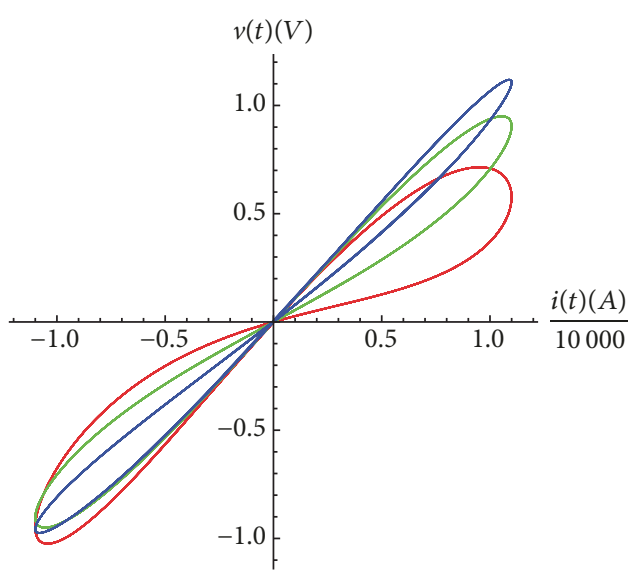

FIGURE 18: $v(t)-i(t)$ of the fractional order memristor at asymptotic state $\left(I_{m}>0\right): \alpha=0.75$ (red), $\alpha=1$ (green), and $\alpha=1.25$ (blue).

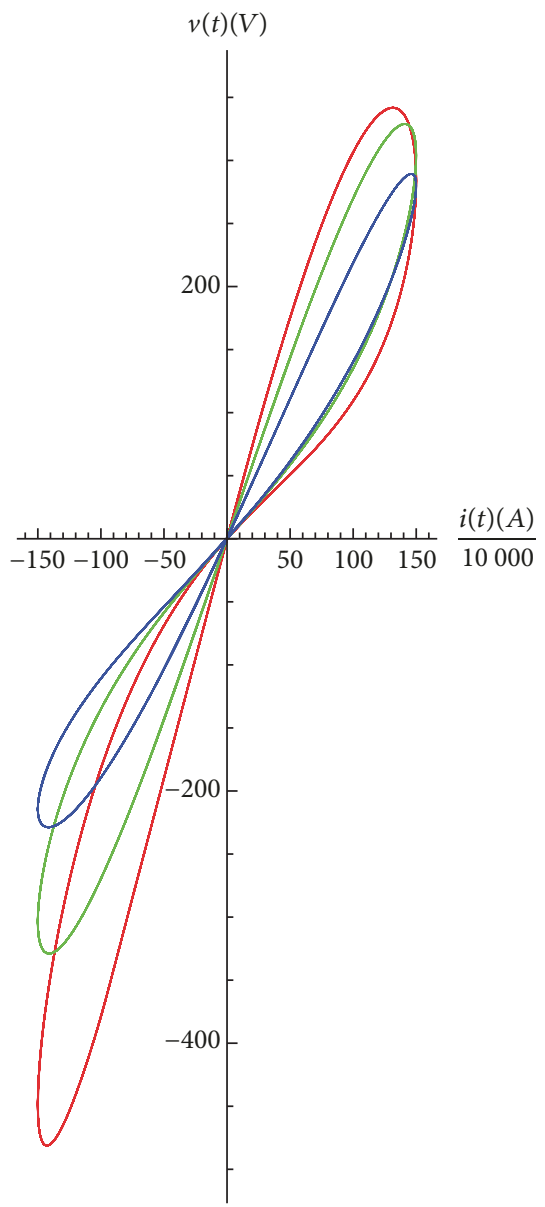

FIGURE 19: $v(t)-i(t)$ of the fractional order memristor at asymptotic state $\left(I_{m}<0\right): \alpha=0.75$ (red), $\alpha=1$ (green), and $\alpha=1.25$ (blue).

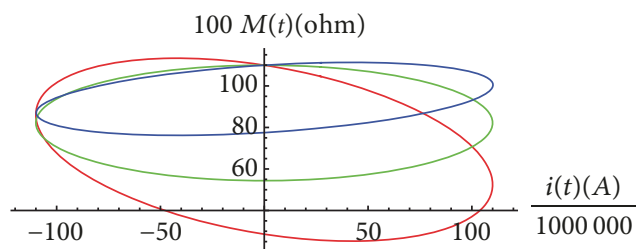

FIgURE 20: $M(t)-i(t)$ of the fractional order memristor $\left(I_{m}>0\right)$ : $\alpha=0.75$ (red), $\alpha=1$ (green), and $\alpha=1.25$ (blue).

$i(t)$. Similarly to the voltage-current curve, the loop area of the memristance-current lissajous curve is also affected by $\alpha$. In particular, the fractional order memristor with lower $\alpha$ yields the memristance-current lissajous curve with wider loop area.

At this point, we will analytically show that both upper and lower lobes of the voltage-current lissajous curve have equal sizes of areas which means that the fractional order memristor does not store the energy, and such areas are independent of $\theta$. Here, we let the area of the upper and lower lobes of the lissajous curve be denoted, respectively, by $A_{U}$ 


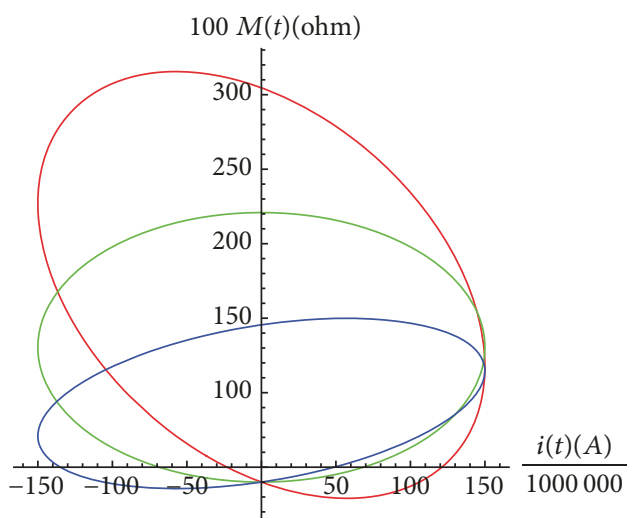

Figure 21: $M(t)-i(t)$ of the fractional order memristor $\left(I_{m}<0\right)$ : $\alpha=0.75$ (red), $\alpha=1$ (green), and $\alpha=1.25$ (blue). and $A_{L}$. Since $v(t)=M_{a}(t) i(t)$ at asymptotic state where $M_{a}(t)$ denotes the asymptotic approximation of $M(t), A_{U}$ and $A_{L}$ can be given by

$$
\begin{aligned}
& A_{U}=\int_{-\theta / \omega}^{(\pi-\theta) / \omega} M_{a}(t) i(t) d i(t) \\
& A_{L}=\int_{(\pi-\theta) / \omega}^{(2 \pi-\theta) / \omega} M_{a}(t) i(t) d i(t)
\end{aligned}
$$

With (13) and the asymptotic approximation of the fractional order integration of sinusoidal function [20], $M_{a}(t)$ can be found as

$$
M_{a}(t)=\min \left[\max \left[M(0)-\frac{K M_{d} I_{m}}{\omega^{\alpha}}\left[\cos \left(\theta+\frac{1-\alpha}{2} \pi\right)-\cos \left(\omega t+\theta+\frac{1-\alpha}{2} \pi\right)\right], M_{o n}\right], M_{o f f}\right]
$$

As $i(t)=I_{m} \sin (\omega t+\theta)$ and the fractional order memristor is unsaturated for the entire simulation period as can be seen from Figures 18 and 19, $A_{U}$ and $A_{L}$ can be finally obtained as follows:

$$
\begin{aligned}
& A_{U}=\frac{2}{3} \frac{K M_{d} I_{m}^{3}}{\omega^{\alpha}} \sin \left(\frac{\alpha \pi}{2}\right) \\
& A_{L}=-\frac{2}{3} \frac{K M_{d} I_{m}^{3}}{\omega^{\alpha}} \sin \left(\frac{\alpha \pi}{2}\right)
\end{aligned}
$$

which show that the upper and lower lobes of the lissajous curve employ equal sizes of areas and such areas are independent of $\theta$. Unlike [20] which considered only $A_{U}$, $A_{L}$ has also been formulated in this work. Moreover, our $A_{U}$ which has been derived by using a different approach from that used in [20] totally agrees with the previous result that $\theta=0 \mathrm{rad}$ has been assumed. This emphasizes the independency from $\theta$ of the lobe areas. Moreover, (24) and (25) also show that the fractional order memristor does not store the energy as the summation of $A_{U}$ and $A_{L}$ which, respectively, referred to the intake and dissipated energy, is equal to 0 .

Besides $A_{U}$ and $A_{L}$, the area within the closed loop of the memristance-current lissajous curve $\left(A_{M}\right)$ can be obtained by also using (23) and

$$
A_{M}=\int_{-\theta / \omega}^{(2 \pi-\theta) / \omega} M_{a}(t) d i(t)
$$

Since it can be seen from Figures 20 and 21 that the device is unsaturated for the whole simulation period and $i(t)=$ $I_{m} \sin (\omega t+\theta)$, we have

$$
A_{M}=\frac{K M_{d} I_{m}^{2} \pi}{\omega^{\alpha}} \sin \left(\frac{\alpha \pi}{2}\right)
$$

which shows that $A_{M}$ is affected by $\alpha$ but independent of $\theta$ as well as $A_{U}$ and $A_{L}$.
Despite the aforementioned independencies, $\theta$ does affect the behavior of the fractional order memristor. For illustration, $M_{a}(t)$ 's due to various $\theta$ 's have been simulated by using (20) as depicted in Figures 22 and 23 where $\alpha=1$ and $\omega=1$ $\mathrm{rad} / \mathrm{sec}$ has been assumed. It should be mentioned here that $I_{m}=110 \mu \mathrm{A}, K=10000 \mathrm{~A}^{-1} \sec ^{-\alpha}, M_{\text {on }}=100 \Omega, M_{\text {off }}=16$ $\mathrm{k} \Omega$, and $M(0)=11 \mathrm{k} \Omega$ have been adopted in Figure 20 where $I_{m}=-150 \mu \mathrm{A}, K=10000 \mathrm{~A}^{-1} \sec ^{-\alpha}, M_{\text {on }}=100 \Omega, M_{\text {off }}=$ $38 \mathrm{k} \Omega$, and $M(0)=11.2 \mathrm{k} \Omega$ have been assumed in Figure 21 . Moreover, $\theta=\pi / 4 \mathrm{rad}, \theta=3 \pi / 4 \mathrm{rad}, \theta=-3 \pi / 4 \mathrm{rad}$, and $\theta=$ $-\pi / 4 \mathrm{rad}$ have been chosen as they are good representatives of those $\theta$ 's which their coordinates on the Euclidian plane, i.e., $(\cos (\theta), \sin (\theta))$, are located on the portion of unit circle's arc in quadrants $1,2,3$, and 4 of such plane, respectively. This is because $(\cos (\theta), \sin (\theta))$ 's of these chosen $\theta$ 's are exactly located at the middle points of the portion of unit circle's arc. For example, $(\cos (\theta), \sin (\theta))$ of $\theta=\pi / 4 \mathrm{rad}$ is located at the middle point of the portion of unit circle's arc in quadrant 1 , etc.

From both figures, it can be seen that these $M_{a}(t)$ 's contain time independent terms which are formerly the time proportional term of $M(t)$ that become time independent at asymptotic state as aforementioned. Since these time independent terms lie within $\left[M_{o n}, M_{o f f}\right], M_{a}(t)$ takes the shape of sinusoidal waveform. However, the minimum peaks of $M_{a}(t)$ 's due to the input with $I_{m}<0$ and $(\cos (\theta), \sin (\theta))$ located on the portion of unit circle's arc in quadrants 2 and 3 of the Euclidian plane have been clipped as can be seen from Figure 23 due to the saturation of the fractional order memristor as these peaks are lower than $M_{\text {on }}$. From Figure 22, it can be seen that the input with $I_{m}>0$ and $\theta$ with $(\cos (\theta), \sin (\theta))$ located on the portion of unit circle's arc in quadrants 2 and 3 yields $M(t)$ with higher time average. If we have assumed that $M_{o n} \leq M_{a}(t) \leq M_{o f f}$ is always satisfied, it can be seen from Figure 23 that $M(t)$ with higher time average can be obtained by using the input with $I_{m}<0$ and $(\cos (\theta), \sin (\theta))$ located on such portion in quadrants 1 and 4 . 


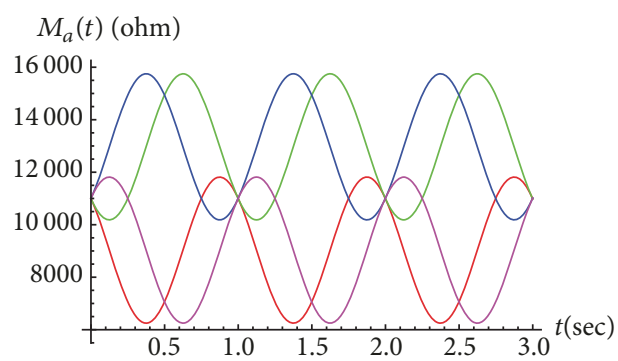

FIGURE 22: $M_{a}(t)$ of the fractional order memristor under the sinusoidal input with $I_{m}>0$ : $\theta=45^{\circ}$ (red), $\theta=135^{\circ}$ (green), $\theta=225^{\circ}$ (blue), and $\theta=315^{\circ}$ (magenta).

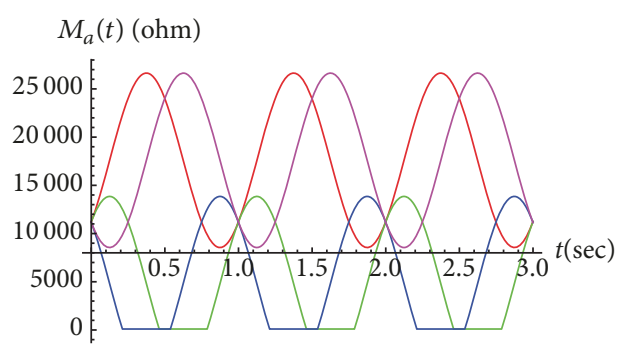

FIgURE 23: $M_{a}(t)$ of the fractional order memristor under the sinusoidal input with $I_{m}<0$ : $\theta=45^{\circ}$ (red), $\theta=135^{\circ}$ (green), $\theta=225^{\circ}$ (blue), and $\theta=315^{\circ}$ (magenta).

Now, we will derive the analytical expression of time independent term of $M_{a}(t)$. Let such term be denoted by $M_{T I}$; it can be given by carefully observing (23) as follows:

$$
\begin{aligned}
& M_{T I} \\
& \quad=\min \left[\max \left[M(0)-\frac{K M_{d} I_{m}}{\omega^{\alpha}}\left[\cos \left(\theta+\frac{1-\alpha}{2} \pi\right)\right], M_{o n}\right],\right. \\
& \left.\quad M_{\text {off }}\right]
\end{aligned}
$$

When $\alpha=1$ as assumed in our simulations of $M_{a}(t), M_{T I}$ can be reduced to

$$
M_{T I}=\min \left[\max \left[M(0)+\Delta M_{T I}, M_{o n}\right], M_{o f f}\right]
$$

where

$$
\Delta M_{T I}=-K \omega^{-1} M_{d} I_{m} \cos (\theta)
$$

Therefore, it can be seen that when $I_{m}>0\left(I_{m}<0\right)$, if $(\cos (\theta), \sin (\theta))$ is located on the portion of unit circle's arc in either quadrant 2 or 3 where $\cos (\theta)<0, \Delta M_{T I}>0\left(\Delta M_{T I}<\right.$ $0)$ thus $M(0)$ has been increased (decreased). On the other hand, if $(\cos (\theta), \sin (\theta))$ is on the portion of unit circle's arc in either quadrant 1 or 4 where $\cos (\theta)>0, \Delta M_{T I}<0$ $\left(\Delta M_{T I}>0\right)$ as $I_{m}>0\left(I_{m}<0\right)$ thus $M(0)$ has been decreased (increased). As a result, the sinusoidal input with $I_{m}>0$ $\left(I_{m}<0\right)$ and $(\cos (\theta), \sin (\theta))$ located on the portion of unit circle's arc in either quadrant 2 or 3 (1 or 4 ) of the Euclidian plane yields higher $M_{T I}$ as graphically observed.

At this point, the influence of $\alpha$ to $M_{T I}$ will be explored. By using (28) with $\theta=0 \mathrm{rad}$ and $\omega=1 \mathrm{rad} / \mathrm{sec}$, we can simulate $M_{T I}$ as shown in Tables 1 and 2. In Table 1, we assume that $I_{m}=110 \mu \mathrm{A}, K=10000 \mathrm{~A}^{-1} \sec ^{-\alpha}, M_{\text {on }}=100 \Omega, M_{\text {off }}=$ $16 \mathrm{k} \Omega$, and $M(0)=11 \mathrm{k} \Omega$, where $I_{m}=-150 \mu \mathrm{A}, K=10000$ $\mathrm{A}^{-1} \sec ^{-\alpha}, M_{\text {on }}=100 \Omega, M_{\text {off }}=38 \mathrm{k} \Omega$, and $M(0)=11.2 \mathrm{k} \Omega$ have been adopted in Table 2 . It can be seen from these tables that $M_{T I}$ is proportional to $\alpha$ when $I_{m}>0$ and vice versa when $I_{m}<0$.

By subtracting $M_{T I}$ from $M_{a}(t)$, we obtain the following purely periodic term of $M(t), M_{P}(t)$

$$
\begin{aligned}
& M_{P}(t) \\
& \quad=\min \left[\max \left[\frac{K M_{d} I_{m}}{\omega^{\alpha}} \cos \left(\omega t+\theta+\frac{1-\alpha}{2} \pi\right), M_{o n}\right],\right. \\
& \left.\quad M_{o f f}\right]
\end{aligned}
$$

which existed in both initial state and asymptotic state.

Therefore, the time proportional term of $M(t), M_{T P}(t)$ can be formulated by subtracting $M_{P}(t)$ from $M(t)$ as

$$
\begin{aligned}
& M_{T P}(t) \\
& =\min \left[\max \left[M(0)-\frac{K M_{d} I_{m} t^{\alpha}}{\Gamma(\alpha+1)}\left[\sin (\theta){ }_{1} \mathrm{~F}_{2}\left(1 ; \frac{\alpha}{2}+\frac{1}{2}, \frac{\alpha}{2}+1 ;-\frac{1}{4}(\omega t)^{2}\right)+\frac{\omega t \cos (\theta)}{\alpha+1}{ }_{1} \mathrm{~F}_{2}\left(1 ; \frac{\alpha}{2}+1, \frac{\alpha}{2}+\frac{3}{2} ;-\frac{1}{4}(\omega t)^{2}\right)\right]-\frac{k M_{d} I_{m}}{\omega^{\alpha}} \cos \left(\omega t+\theta+\frac{1-\alpha}{2} \pi\right), M_{o n}\right],\right. \\
& \left.M_{\text {off }}\right]
\end{aligned}
$$

As a result, the aforementioned $T_{\text {sat }}$ can be determined by solving the following equation:

$$
\begin{aligned}
& \left.\begin{array}{ll}
M_{o n} & I_{m}>0 \\
M_{\text {off }} & I_{m}<0
\end{array}\right\}=M(0)-\frac{K M_{d} I_{m} T_{s a t}^{\alpha}}{\Gamma(\alpha+1)}[\sin (\theta) \\
& \cdot{ }_{1} \mathrm{~F}_{2}\left(1 ; \frac{\alpha}{2}+\frac{1}{2}, \frac{\alpha}{2}+1 ;-\frac{1}{4}\left(\omega T_{\text {sat }}\right)^{2}\right)
\end{aligned}
$$


TABLE 1: $M_{T I}$ due to $I_{M}>0$.

\begin{tabular}{lc}
\hline$\alpha$ & $M_{T I}(\mathrm{k} \Omega)$ \\
\hline 0.75 & 6.9296 \\
1.00 & 8.2175 \\
1.25 & 9.7365 \\
\hline
\end{tabular}

TABLE 2: $M_{T I}$ due to $I_{M}<0$.

\begin{tabular}{lc}
\hline$\alpha$ & $M_{T I}(\mathrm{k} \Omega)$ \\
\hline 0.75 & 24.4306 \\
1.00 & 20.2443 \\
1.25 & 16.4772 \\
\hline
\end{tabular}

At this point, we will derive the expressions of $M(t)$ 's due to other $\mathrm{AC}$ waveforms by using that due to the sinusoidal waveform, i.e., (19), as the basis. For example, the expression of $M(t)$ due to arbitrary phase cosinusoidal waveform, i.e., $i(t)=I_{m} \cos (\omega t+\theta)$, can be formulated by using (19) and the relationship between the sinusoidal and cosinusoidal functions is given by $\cos (u)=$ $\sin ((\pi / 2)-u)$ where $u$ denotes arbitrary angle. As a result, we have

$$
\begin{aligned}
& M(t) \\
& \quad=\min \left[\max \left[M(0)-\frac{K M_{d} I_{m} t^{\alpha}}{\Gamma(\alpha+1)}\left[\sin (\theta){ }_{1} \mathrm{~F}_{2}\left(1 ; \frac{\alpha}{2}+\frac{1}{2}, \frac{\alpha}{2}+1 ;-\frac{1}{4}\left(\frac{\pi}{2}-\omega t-2 \theta\right)^{2}\right)+\frac{(\pi / 2-\omega t-2 \theta) \cos (\theta)}{\alpha+1}{ }_{1} \mathrm{~F}_{2}\left(1 ; \frac{\alpha}{2}+1, \frac{\alpha}{2}+\frac{3}{2} ;-\frac{1}{4}\left(\frac{\pi}{2}-\omega t-2 \theta\right)^{2}\right)\right], M_{o n}\right],\right. \\
& \left.M_{\text {off }}\right]
\end{aligned}
$$

For arbitrary periodic waveform which has never been considered in those previous works, the resulting expression can also be determined based on (19). This is because such waveform can be given as a series of sinusoidal functions, i.e., $i(t)=\sum_{n=0}^{\infty}\left[I_{m n} \sin \left(n \omega t+\theta_{n}\right)\right]$, where $I_{m n}$ and $\theta_{n}$, respectively, stand for peak value and phase of arbitrary $n$th term of the series, according to the Fourier theorem. As a result, the expression of $M(t)$ due to arbitrary periodic waveform can be given as follows:

$$
\begin{aligned}
& M(t) \\
& \quad=\min \left[\max \left[M(0)-\frac{K M_{d} t^{\alpha}}{\Gamma(\alpha+1)} \sum_{n=0}^{\infty}\left[I_{m n}\left[\sin \left(\theta_{n}\right){ }_{1} \mathrm{~F}_{2}\left(1 ; \frac{\alpha}{2}+\frac{1}{2}, \frac{\alpha}{2}+1 ;-\frac{1}{4}(n \omega t)^{2}\right)+\frac{n \omega t \cos \left(\theta_{n}\right)}{\alpha+1}{ }_{1} \mathrm{~F}_{2}\left(1 ; \frac{\alpha}{2}+1, \frac{\alpha}{2}+\frac{3}{2} ;-\frac{1}{4}(n \omega t)^{2}\right)\right]\right], M_{o n}\right],\right. \\
& \left.\quad M_{o f f}\right]
\end{aligned}
$$

\section{The Usage of Fractional Order Memristor in the Memristor Based Circuit}

In this research, the HP memristor based type A Wien oscillator [29] has been chosen as the candidate memristor based circuit. The unique characteristic of such circuit, which is either $R_{1}$ or $R_{2}$ replaced by the memristive device as depicted in Figure 22, is the fluctuated frequency of oscillation. The smaller range of fluctuation refers to the better chance that the system has sustained oscillation which can be obtained if and only if all poles of the system are fixed in the s-plane [29]. For studying the usage of fractional order memristor, we replace $R_{1}$ of the circuit by such fractional order device instead of the HP memristor as traditionally did [29] and analyze the effect of $\alpha$ to the fluctuation in frequency of oscillation which determines the chance that the system has sustained oscillation, as mentioned above. Let the range of such fluctuation be denoted by $\Delta f_{\text {osc }}$; it can be mathematically defined as given by (36) where $f_{\text {up }}$ and $f_{\text {low }}$ stand for the upper bound and lower boundary.

$$
\Delta f_{\text {osc }}=f_{\text {up }}-f_{\text {low }}
$$

According to [29], these boundaries can be obtained by solving (37) and (38) which have been formulated by assuming that $C_{1}=C_{2}=C$, the memristor is unsaturated, and the frequency of oscillation has been found to lie within the range that the sustained oscillation can be assured. Note also that $I_{\text {mem }}=V_{\text {mem }} / M(0)$, where $V_{\text {mem }}$ denotes the peak value of the voltage dropped across the memristor which can be determined from the oscillator output voltage, $V_{\text {out }}(t)$, and also depends on the initial voltages of $C_{1}$ and $C_{2}$ [29]. Moreover, $V_{\text {out }}(t)$ can be obtained from the state space representation and output equation of the fractional memristor based Wien oscillator which are, respectively, given by (39) and (40) where $V_{C 1}(t)$ and $V_{C 2}(t)$ denote the voltage dropped across $C_{1}$ and $C_{2}$ and $M(t)$ stands for the memristance of the conventional HP memristor.

$$
\begin{aligned}
& f_{u p} \\
& =\sqrt{\frac{4}{16 \pi^{2} C^{2}}-\frac{\left(k M_{d}\left|I_{\text {mem }}\right| / 2 \pi f_{u p}\right)^{2}}{16 \pi^{2} C^{2}\left(M(0)-k M_{d}\left|I_{\text {mem }}\right| / 2 \pi f_{u p}\right) R_{2}}}
\end{aligned}
$$




$$
\begin{aligned}
& f_{\text {low }} \\
& =\sqrt{\frac{4}{16 \pi^{2} C^{2}}-\frac{\left(k M_{d}\left|I_{\text {mem }}\right| / 2 \pi f_{\text {low }}\right)^{2}}{16 \pi^{2} C^{2}\left(M(0)+k M_{d}\left|I_{\text {mem }}\right| / 2 \pi f_{\text {low }}\right) R_{2}}} \\
& {\left[\begin{array}{l}
\frac{d}{d t} V_{C_{1}}(t) \\
\frac{d}{d t} V_{C_{2}}(t)
\end{array}\right]} \\
& =\left[\begin{array}{cc}
-\frac{1}{M(t) C_{1}} & \frac{R_{3}}{R_{4} M(t) C_{1}} \\
-\frac{1}{M(t) C_{2}} & \frac{R_{3}}{R_{4} M(t) C_{2}}-\frac{1}{R_{2} C_{2}}
\end{array}\right]\left[\begin{array}{c}
V_{C_{1}}(t) \\
V_{C_{2}}(t)
\end{array}\right] \\
& V_{\text {out }}(t)=\left(\frac{R_{3}}{R_{4}}+1\right) V_{C_{2}}(t)
\end{aligned}
$$

However, this is not the case when the fractional order memristor has been used as $M(t)$ of such device must be adopted. Therefore $V_{\text {out }}(t)$ must be determined based on our derived $M(t)$ instead where $f_{u p}$ and $f_{\text {low }}$ must be evaluated from (41) and (42). As a result, the corresponding $\Delta f_{\text {osc }}$ will be different from that of the original conventional memristor based circuit and the different chance of obtaining sustained oscillation can be expected.

$$
\begin{aligned}
& f_{\text {up }} \\
& =\sqrt{\frac{4}{16 \pi^{2} C^{2}}-\frac{\left(K M_{d}\left|I_{\text {mem }}\right| /\left(2 \pi f_{\text {up }}\right)^{\alpha}\right)^{2}}{16 \pi^{2} C^{2}\left(M(0)-K M_{d}\left|I_{\text {mem }}\right| /\left(2 \pi f_{\text {up }}\right)^{\alpha}\right) R_{2}}} \\
& f_{\text {low }} \\
& =\sqrt{\frac{4}{16 \pi^{2} C^{2}}-\frac{\left(K M_{d}\left|I_{\text {mem }}\right| /\left(2 \pi f_{\text {low }}\right)^{\alpha}\right)^{2}}{16 \pi^{2} C^{2}\left(M(0)+K M_{d}\left|I_{\text {mem }}\right| /\left(2 \pi f_{\text {low }}\right)^{\alpha}\right) R_{2}}}
\end{aligned}
$$

Moreover, the condition for ensuring the occurrence of sustained oscillation can be given by () where $f_{\text {avr }}$ which stands for the average oscillating frequency is given by ().

$$
\begin{aligned}
& \frac{R_{3}}{R_{4}}=1+\frac{1}{R_{2}}\left[M(0)-\frac{K M_{d}\left|I_{\text {mem }}\right|}{\left(2 \pi f_{\text {avr }}\right)^{\alpha}}\right] \\
& f_{\text {avr }} \\
& =\sqrt{\frac{4}{16 \pi^{2} C^{2}}-\frac{\left(K M_{d}\left|I_{\text {mem }}\right| /\left(2 \pi f_{\text {avr }}\right)^{\alpha}\right)^{2}}{16 \pi^{2} C^{2}\left(M(0)-K M_{d}\left|I_{\text {mem }}\right| /\left(2 \pi f_{\text {avr }}\right)^{\alpha}\right) R_{2}}}
\end{aligned}
$$

By assuming $0.1 \mathrm{~V}$ and $-0.95 \mathrm{~V}$ as the approximate initial voltages of $C_{1}$ and $C_{2}$ and also assuming that $R_{2}=5 \mathrm{k} \Omega, C_{1}=$ $C_{2}=3.2 \mu \mathrm{F}, K=100000 \mathrm{~A}^{-1} \sec ^{-\alpha}, M_{\text {on }}=100 \Omega$, and $M_{\text {off }}$ $=16 \mathrm{k} \Omega, \Delta f_{\text {osc }}$ of the fractional order memristor based Wien oscillator with $\alpha<1$ and $\alpha>1$ can be obtained for various $M(0)$ 's by numerically solving (41) and (42). as tabulated in Table 3 where $\Delta f_{\text {osc }}$ of the original HP memristor based circuit which is equivalent to the fractional order memristor with $\alpha=1$ in the context of this work, determined by solving (37) and (38), has also been included.
TABLE 3: $\Delta f_{\text {osc }}(\mathrm{Hz})$ for various $M(0)$ 's.

\begin{tabular}{lccc}
\hline$M(0)(\mathrm{k} \Omega)$ & $\alpha=0.75$ & $\mathrm{HP}(\alpha=1)$ & $\alpha=1.25$ \\
\hline 4.1 & 9.16 & 3.32 & 1.49 \\
4.4 & 7.64 & 2.88 & 1.32 \\
4.7 & 6.44 & 2.52 & 1.19 \\
5.0 & 5.49 & 2.23 & 1.07 \\
5.3 & 4.73 & 1.98 & 0.97 \\
5.6 & 4.11 & 1.78 & 0.89 \\
5.9 & 3.59 & 1.60 & 0.81 \\
\hline
\end{tabular}

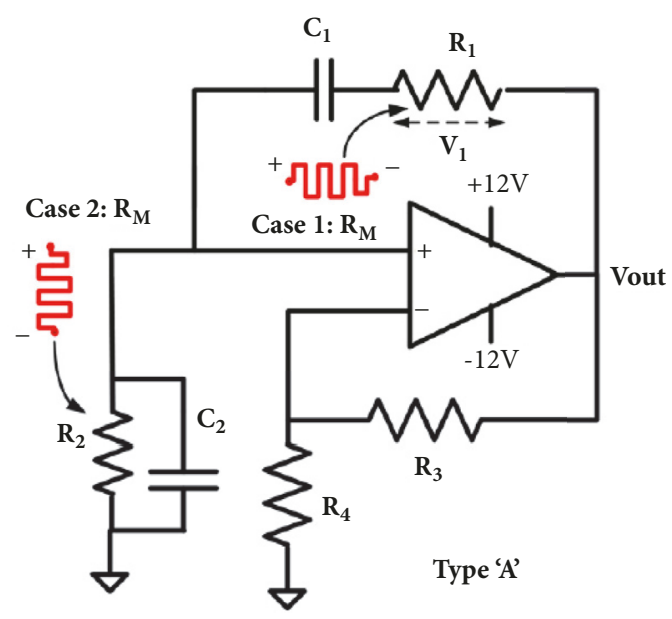

Figure 24: The memristor based Wien oscillator [29].

It has been found that $\Delta f_{\text {osc }}$ is inversely proportional to $M(0)$ which is in agreement with [29]. Since $4.1 \mathrm{k} \Omega \leq$ $M(0) \leq 5.9 \mathrm{k} \Omega$ [29] and the probability of obtaining sustained oscillation is inversely proportional to $\Delta f_{\text {osc }}, M(0)$ $=5.9 \mathrm{k} \Omega$ is recommended as it minimizes $\Delta f_{\text {osc }}$ and thus maximizes such probability. It can also be seen that $\Delta f_{\text {osc }}$ is inversely proportional to $\alpha$ which means that the probability of obtaining sustained oscillation is directly proportional to $\alpha$. Therefore, the fractional order memristor with larger $\alpha$ is recommended and the fractional order memristor with $\alpha>1$ should be adopted as it can increase such probability from that of the original circuit which its memristive device has $\alpha=1$. On the other hand, the fractional order memristor with $\alpha<1$ should be avoided as it decreases such probability.

Finally, by further assuming that $M(0)=5 \mathrm{k} \Omega, R_{3}=20.2$ $\mathrm{k} \Omega$, and $R_{4}=10 \mathrm{k} \Omega$, we can simulate $V_{\text {out }}(t)$ at asymptotic state and the lissajous patterns of $V_{\text {out }}(t)$ and $M(t)$ for various $\alpha$ 's as depicted in Figures 23-28 where the units of $100 V_{\text {out }}(t)$, $t$, and $M(\mathbf{t})$ are $\mathrm{V}$, sec, and $\Omega$, respectively. The comparison of the results with $\alpha=1$ to the SPICE HP memristor model based counterparts has been made for verification where a strong agreement can be observed. We have found that there exists neither temporary nor permanent saturation of the fractional order memristor. This is because $M(t)$ is always within $\left[M_{o n}, M_{o f f}\right]$ in this scenario due to the assumed conditions on parameters and input, as can be seen from the simulated lissajous patterns. Such conditions have been adopted for ensuring the unsaturation which yields the 


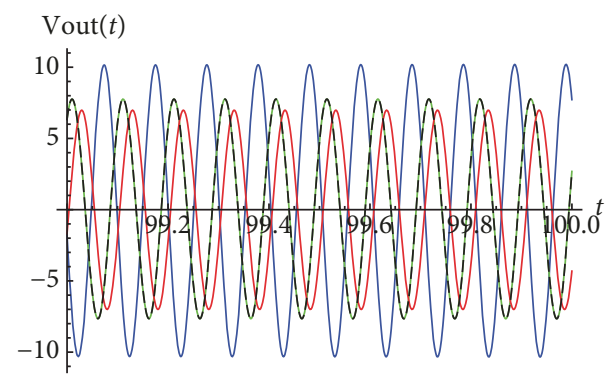

FIgURE 25: $V_{\text {out }}(t)$ at asymptotic state of Type A Wien oscillator: fractional memristor with $\alpha=0.75$ (blue), fractional memristor with $\alpha=1$ (green), fractional memristor with $\alpha=1.25$ (red), and HP memristor (black dots).

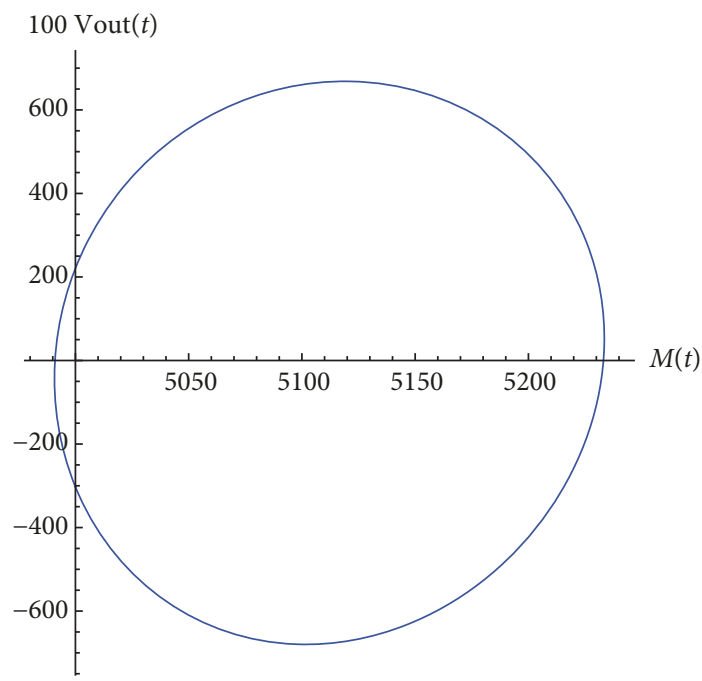

FIgURE 26: $V_{\text {out }}(t)$ versus $M(t)$ of Type A Wien oscillator: fractional memristor with $\alpha=0.75$.

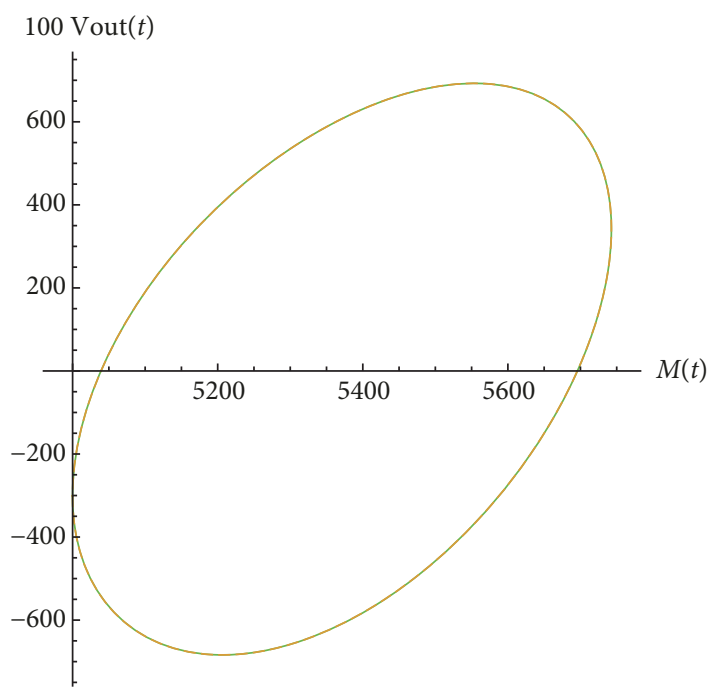

FIGURE 27: $V_{\text {out }}(t)$ versus $M(t)$ of Type A Wien oscillator: fractional memristor with $\alpha=1$ (green line) and HP memristor (orange dashed).

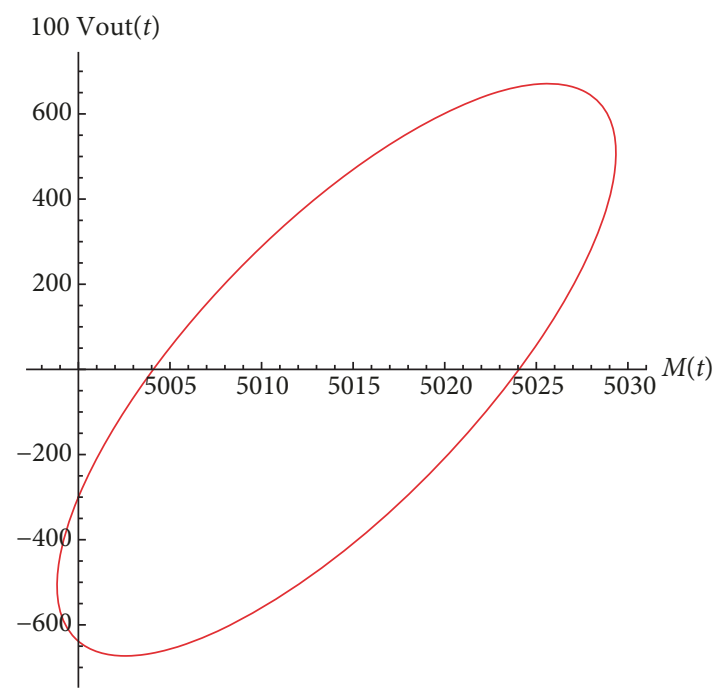

FIGURE 28: $V_{\text {out }}(t)$ versus $M(t)$ of Type A Wien oscillator: fractional memristor with $\alpha=1.25$.

proper operation of the oscillator [29]. From these lissajous patterns, we have also found that $V_{\text {out }}(t)$ is of the same frequency as $M(t)$ and the phase difference between $V_{\text {out }}(t)$ and $M(t)$, which is less than $90^{\circ}$, is inversely proportional to $\alpha$.

\section{Conclusion}

In this work, the HP memristor has been generalized in a fractional order domain by applying the fractional calculus to its state equation. Unlike [18-21], the dimensional consistency has been taken into account. Moreover, the boundary effect has also been modelled. Therefore the analytical expression of $M(t)$ which has been derived as a function of $i(t)$ and its related results are dimensional consistent and valid to such generalized device in its saturation states. By using such expression, $M(t)$ 's due to various waveforms and the related parameters have been formulated. With the simulations by using these $M(t)$ 's and parameters, the behaviors of the fractional order memristor have been thoroughly explored. Therefore this research gives a precise understanding on the characteristics of such up to date nonlinear electrical circuit element which has been recently applied as the basis of the net grid type fracmemristor [31].

\section{Data Availability}

The simulated data used to support the findings of this study are included within the article.

\section{Conflicts of Interest}

The author declares that there are no conflicts of interest regarding the publication of this article. 


\section{Acknowledgments}

The author would like to acknowledge Mahidol University, Thailand, for the online database service which is our primary information resource.

\section{References}

[1] L. O. Chua, "Memristor-the missing circuit element," IEEE Transactions on Circuit Theory, vol. 18, no. 5, pp. 507-519, 1971.

[2] I. S. Jesus, J. A. Tenreiro MacHado, and J. Boaventure Cunha, "Fractional electrical impedances in botanical elements," Journal of Vibration and Control, vol. 14, no. 9-10, pp. 1389-1402, 2008.

[3] C. Tang, F. You, G. Cheng, D. Gao, F. Fu, and X. Dong, "Modeling the frequency dependence of the electrical properties of the live human skull," Physiological Measurement, vol. 30, no. 12, pp. 1293-1301, 2009.

[4] A. Charef, "Analogue realisation of fractional-order integrator, differentiator and fractional $\operatorname{PI} \lambda \mathrm{D} \mu$ controller," IEE Proceedings-Control Theory and Applications, vol. 153, no. 6, pp. 714-720, 2006.

[5] B. M. Vinagre and V. Feliu, "Optimal fractional controllers for rational order systems: a special case of the Wiener-Hopf spectral factorization method," Institute of Electrical and Electronics Engineers Transactions on Automatic Control, vol. 52, no. 12, pp. 2385-2389, 2007.

[6] R. Matusu, "Application of fractional order calculus to control theory," International Journal of Mathematical Models and Methods in Applied Sciences, vol. 5, pp. 1162-1169, 2011.

[7] L. Dork, J. Terpk, I. Petr, and F. Dorkov, "Electronic realization of the fractional-order systems," Acta Montanistica Slovaca, vol. 12, pp. 231-237, 2007.

[8] B. T. Krishna, K. V. V. S. Reddy, and S. Santha Kumari, "Time domain response calculations of fractance device of order 1/2," Journal of Active \& Passive Electronic Devices, vol. 3, pp. 355-367, 2008.

[9] A. G. Radwan and A. S. Elwakil, "An expression for the voltage response of a current-excited fractance device based on fractional-order trigonometric identities," International Journal of Circuit Theory and Applications, vol. 40, no. 5, pp. 533-538, 2012.

[10] D. B. Strukov, G. S. Snider, D. R. Stewart, and R. S. Williams, "The missing memristor found," Nature, vol. 453, pp. 80-83, 2008.

[11] A. G. Radwan, M. A. Zidan, and K. N. Salama, "HP Memristor mathematical model for periodic signals and DC," in Proceedings of the 2010 53rd IEEE International Midwest Symposium on Circuits and Systems (MWSCAS), pp. 861-864, Seattle, Wash, USA, August 2010.

[12] A. G. Radwan, M. A. Zidan, and K. N. Salama, "On the mathematical modeling of Memristors," in Proceedings of the 2010 International Conference on Microelectronics, ICM'10, pp. 284-287, Cairo, Egypt, December 2010.

[13] Y. N. Joglekar and S. J. Wolf, "The elusive memristor: properties of basic electrical circuits," European Journal of Physics, vol. 30, no. 4, pp. 661-675, 2009.

[14] S. Shin, K. Kim, and S.-M. Kang, "Compact models for memristors based on charge-flux constitutive relationships," IEEE Transactions on Computer-Aided Design of Integrated Circuits and Systems, vol. 29, no. 4, pp. 590-598, 2010.
[15] C. Yakopcic, T. M. Taha, G. Subramanyam, R. E. Pino, and S. Rogers, "A memristor device model," IEEE Electron Device Letters, vol. 32, no. 10, pp. 1436-1438, 2011.

[16] J. Tenreiro Machado, "Fractional generalization of memristor and higher order elements," Communications in Nonlinear Science and Numerical Simulation, vol. 18, no. 2, pp. 264-275, 2013.

[17] Y.-F. Pu and X. Yuan, "Fracmemristor: fractional-order memristor," IEEE Access, vol. 4, pp. 1872-1888, 2016.

[18] M. E. Fouda and A. G. Radwan, "On the fractional-order memristor model," Fractional Calculus and Applied Analysis, vol. 4, pp. 1-7, 2013.

[19] M. E. Fouda and A. G. Radwan, "Fractional-order Memristor Response Under DC and Periodic Signals," Circuits, Systems and Signal Processing, vol. 34, no. 3, pp. 961-970, 2015.

[20] Y. Yu, B. Bao, H. Kang, and M. Shi, "Calculating area of fractional-order memristor pinched hysteresis loop," The Journal of Engineering, vol. 2015, no. 11, pp. 325-327, 2015.

[21] M. Shi and S. Hu, "Pinched hysteresis loop characteristics of a fractional-order $\mathrm{HP}^{\mathrm{TiO}_{2}}$ memristor," in Intelligent Computing, Networked Control, and Their Engineering Applications, vol. 762 of Communications in Computer and Information Science, pp. 705-713, Springer, Singapore, Singapore, 2017.

[22] J. F. Gómez-Aguilar, J. J. Rosales-García, J. J Bernal-Alvarado, T. Córdova-Fraga, and R. Guzmán-Cabrera, "Fractional mechanical oscillators," Revista Mexicana de Física, vol. 58, pp. 348-352, 2012.

[23] R. Banchuin, "Novel expressions for time domain responses of fractance device," Cogent Engineering, vol. 4, no. 1, 2017.

[24] A. G. Radwan and E. Mohammed, "Memristor: models, types, and applications," in On the Mathematical Modeling of Memristor, Memcapacitor, and Meminductor, pp. 13-49, Springer, Cham, Switzerland, 2015.

[25] J. Sabatier, O. P. Agrawal, and J. A. Machado, Advance in Fractional Calculus: Theoretical Developments and Applications in Physics and Engineering, Springer, Dordrecht, The Netherlands, 2007.

[26] W. H. Beyer, CRC Handbook of Mathematical Sciences, CRC Press, Boca Raton, Fla, USA, 1987.

[27] Z. Biolek, D. Biolek, and V. Biolková, "SPICE model of memristor with nonlinear dopant drift," Radioengineering, vol. 18, no. 2, pp. 210-214, 2009.

[28] B. Dwork, Generalized Hypergeometric Functions, Clarendon Press, Oxford, UK, 1990.

[29] A. Talukdar, A. G. Radwan, and K. N. Salama, "Generalized model for Memristor-based Wien family oscillators," Microelectronics Journal, vol. 42, no. 9, pp. 1032-1038, 2011.

[30] L. Chua, "If it's pinched it's a memristor," Semiconductor Science and Technology, vol. 29, no. 10, Article ID 104001, 2014.

[31] L. Xu, G. Huang, and Y. Pu, "Numerical Simulation Research of Fracmemristor Circuit Based on HP Memristor," Journal of Circuits, Systems and Computers, vol. 27, no. 14, Article ID 1850227, 2018. 


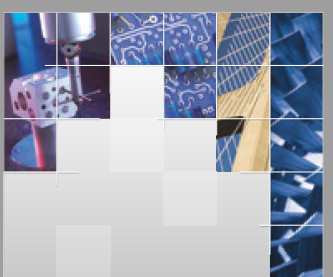

\section{Enfincering}
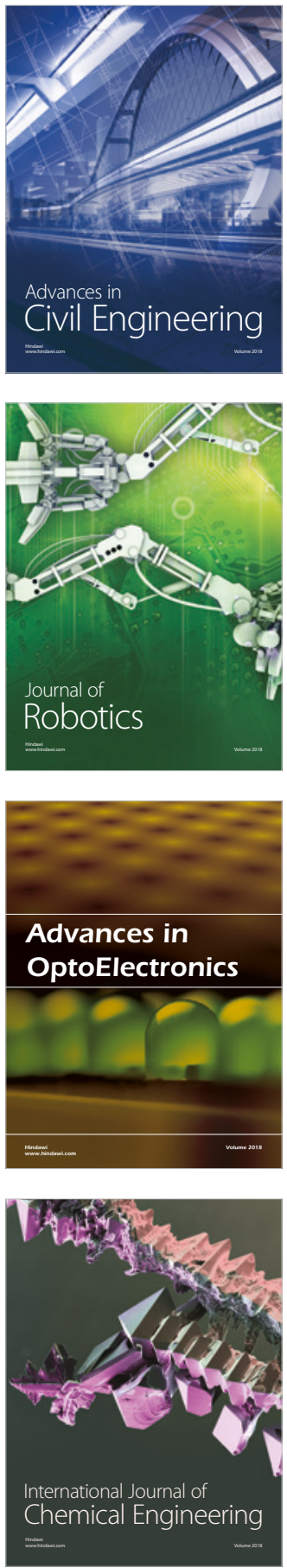

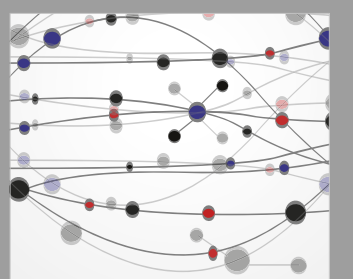

\section{Rotating \\ Machinery}

The Scientific World Journal

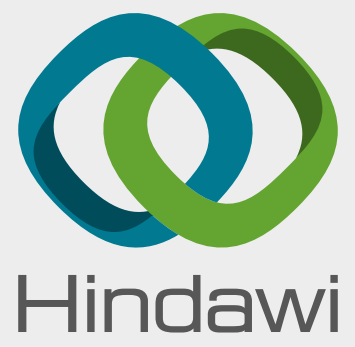

Submit your manuscripts at

www.hindawi.com
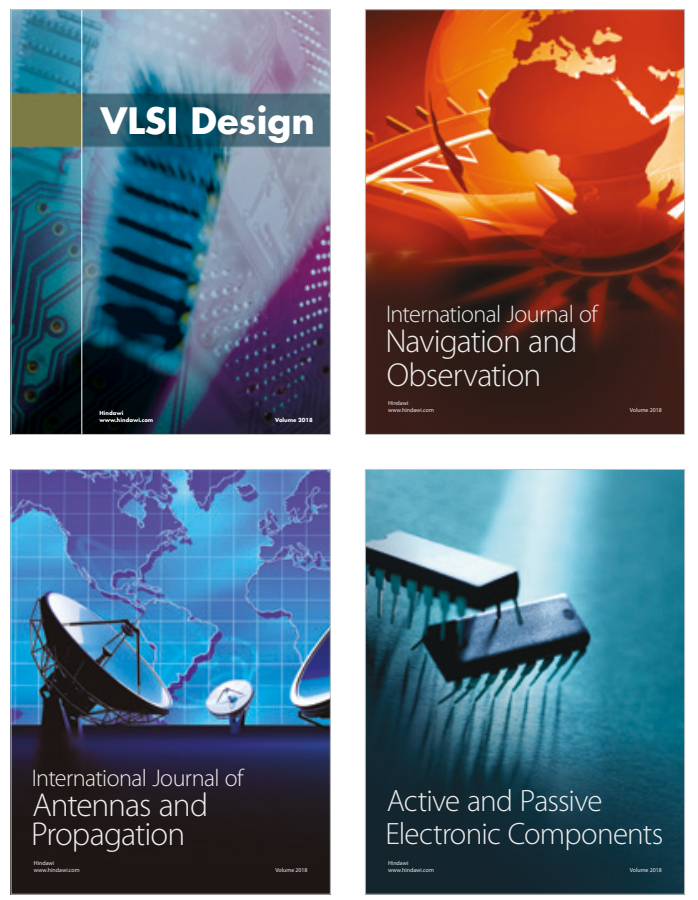
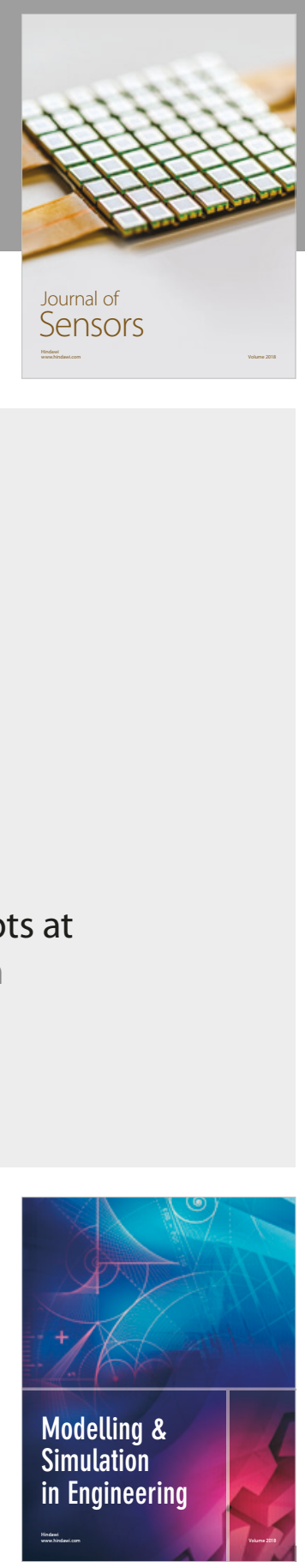

\section{Advances \\ Multimedia}
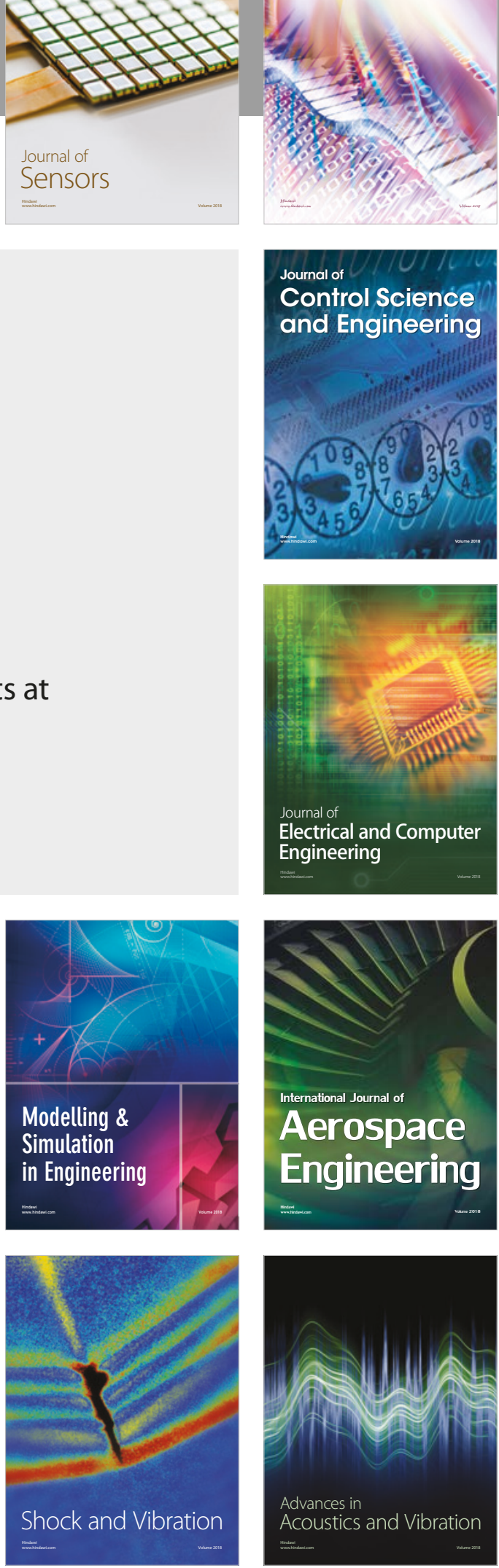